\title{
Gas flow models in the Milky Way embedded bars ${ }^{\star}$
}

\author{
N. J. Rodriguez-Fernandez ${ }^{1}$ and F. Combes ${ }^{2}$ \\ 1 IRAM, 300 rue de la Piscine, 38406 St. Martin d'Hères, France \\ e-mail: rodriguez@iram.fr \\ 2 LERMA, Observatoire de Paris, 61 Av de l'Observatoire, 75014 Paris, France
}

Received 25 February 2008 / Accepted 26 June 2008

ABSTRACT

\begin{abstract}
Context. The gas distribution and dynamics in the inner Galaxy present many unknowns, such as the origin of the asymmetry of the $l v$-diagram of the Central Molecular Zone (CMZ). On the other hand, there is recent evidence in the stellar component of the presence of a nuclear bar that may be slightly lopsided.

Aims. Our goal is to characterize the nuclear bar observed in 2MASS maps and to study the gas dynamics in the inner Milky Way taking into account this secondary bar.

Methods. We have derived a realistic mass distribution by fitting the 2MASS star count map with a model including three components (disk, bulge and nuclear bar) and we have simulated the gas dynamics in the deduced gravitational potential using a sticky-particles code.

Results. Our simulations of the gas dynamics successfully reproduce the main characteristics of the Milky Way for a bulge orientation of $20^{\circ}-35^{\circ}$ with respect to the Sun-Galactic Center (GC) line and a pattern speed of $30-40 \mathrm{~km} \mathrm{~s}^{-1} \mathrm{kpc}^{-1}$. In our models the Galactic Molecular Ring (GMR) is not an actual ring but the inner parts of the spiral arms, while the 3-kpc arm and its far side counterpart are lateral arms that extend around the bar. Our simulations reproduce, for the first time, the parallelogram shape of the $l v$-diagram of the $\mathrm{CMZ}$ as the gas response to the nuclear bar. This bar should be oriented by an angle of $\sim 60^{\circ}-75^{\circ}$ with respect to the Sun-GC line and its mass amounts to (2-5.5) $10^{9} M_{\odot}$. We show that the observed asymmetry of the CMZ cannot be due to lopsidedness of the nuclear bar as suggested by the 2MASS maps.

Conclusions. We do not find clear evidence of lopsidedness in the stellar potential. We propose that the observed asymmetry of the central gas layer can be due to the infalling of gas into the $\mathrm{CMZ}$ in the $l=1.3^{\circ}$-complex.
\end{abstract}

Key words. Galaxy: structure - Galaxy: center - Galaxy: kinematics and dynamics - ISM: kinematics and dynamics methods: numerical

\section{Introduction}

Studying the structure and the dynamics of the Milky Way galaxy is necessary to understand its formation and evolution. For example, the large scale star formation rate, the gas transfer to the nucleus and the fueling of the central super-massive black hole are strongly influenced by the large scale dynamics of the Galaxy.

At least three components are needed to explain the rotation curve of the Galaxy: a disc, a bulge and a massive dark halo. In near-infrared images, the bulge is a triaxial structure difficult to distinguish from a bar. The first direct evidence of the barred nature of the Milky Way was found by Blitz \& Spergel (1991). The analysis of COBE/DIRBE data (Dwek et al. 1995; Binney et al. 1997; Freudenreich 1998) supported Blitz \& Spergel results. Dwek et al. (1995) found that the peanut-shaped bulge seen in the COBE image is best fitted by boxy Gaussians functions. Since COBE, direct evidence of the bar has increased based on photometric surveys and star counts in the near-infrared (see for instance López-Corredoira et al. 2000, 2005; Babusiaux \& Gilmore 2005, and references therein). Although there is a consensus on the triaxial nature of the bulge, the actual shape and the inclination is still under debate with currently no clear agreement among different authors. The inclination angles found

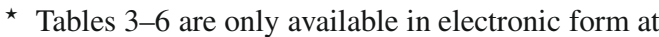
http://www . aanda. org range from $\sim 10^{\circ}-15^{\circ}$ (Binney et al. 1991; Freudenreich 1998; Lopez-Corredoira et al. 1997) to $\sim 30^{\circ}-45^{\circ}$ (Weiner \& Sellwood 1999; Sevenster 1999; López-Corredoira et al. 2001) with a number of works giving values in the range $15^{\circ}-30^{\circ}$ (Mulder \& Liem 1986; Binney et al. 1997; Fux 1999; Englmaier \& Gerhard 1999; Bissantz \& Gerhard 2002; Bissantz et al. 2003; Babusiaux \& Gilmore 2005; López-Corredoira et al. 2005).

In addition to the bar-like boxy-bulge, some recent works have also proposed the existence of another bar that would be longer and thinner than the bulge (Picaud et al. 2003; LópezCorredoira et al. 2001, 2007; Benjamin et al. 2005). As with the bulge, the near side of this long and thin bar would also be located in the first Galactic quadrant. In contrast, the long bar would be oriented at an angle of $\sim 45^{\circ}$ with respect to the line of sight towards Galactic center. In the present paper we will not discuss this component as the mass should be dominated by the bulge. Hereafter, we will refer to the triaxial boxy bulge as the "large bar" or simply "bulge".

Due to the difficulty of measuring stellar velocities, most of our knowledge of the Galactic dynamics comes from spectroscopic observations of the atomic and molecular interstellar medium (Rougoor \& Oort 1960; Scoville 1972; Liszt \& Burton 1978; Bally et al. 1987; Dame et al. 2001). The gas exhibit large non-circular motion in the inner Galaxy. Among the possible explanations for these kinematics, de Vaucouleurs (1964) proposed a gas response to a bar potential. This explanation has also 
been discussed in other observational works such as those of Kerr (1967); Bania (1977) or Liszt \& Burton (1978). Nevertheless, it is the comparison of the observations with numerical simulations of the gas dynamics that have allowed us to better understand the structure of the Galaxy and to derive important dynamical information, such as the pattern speed of the bar. The first attempt was that of Mulder \& Liem (1986) solving the gas-dynamical equations to obtain the gas flow in a weak bar for the whole Galaxy.

A major step in our understanding of the gas kinematics in the Galactic Center was the paper by Binney et al. (1991). These authors compared of the $\mathrm{CO}(1-0)$ and $\mathrm{HI}$ longitude-velocity diagrams (hereafter $l v$-diagram) of Bally et al. (1987) and Burton \& Liszt (1978), with the $l v$ locus of closed stellar orbits in a bar potential ( $X_{1}$ and $X_{2}$ orbits). The $l v$-diagram of the molecular gas in the inner $\sim 4^{\circ}$ of the Galaxy shows a parallelogram shape that resembles the $l v$ locus of the cusped $X_{1}$ orbit for a bar with an inclination of $\sim 16^{\circ}$. In spite of the globally successful interpretation of the CO and HI $l v$-diagrams, the Binney et al. (1991) model cannot account for the observed asymmetry of the $\mathrm{CO}$ parallelogram, which is lopsided towards positive longitudes while the modeled parallelograms are centered at longitude $0^{\circ}$. In addition the expected upper right vertex of the locus of the cusped $X_{1}$ orbit has a velocity close to 0 , while the upper right vertex of the observed CO parallelogram shown in Fig. 2 of Binney et al. (1991) has a velocity of $\sim 150 \mathrm{~km} \mathrm{~s}^{-1}$.

The qualitative insight into the gas dynamics that one could obtain by comparing the $l v$-diagram of the gas with that of a few stellar orbits should be tested by performing simulations of the gas dynamics that take into account the dissipative nature of the gas clouds. This was done by Jenkins \& Binney (1994), who tried to reproduce the $l v$-diagram of the CMZ using a stickyparticles code but with moderate success. Using a combination of SPH and collisionless particles, Lee et al. (1999) were able to reproduce an inner ring with the right inclination in the $l v$-diagram and also some of the gas clouds with non-circular velocities in the range $|l|<6^{\circ}$.

To our knowledge, the works by Binney et al. (1991); Jenkins \& Binney (1994) and Lee et al. (1999) are the only dynamical models that have tried to interpret the dynamics of the complex Galactic center region (the central $500 \mathrm{pc}$ ). Other Galactic dynamics models have been mainly devoted to larger scales, from the works of Mulder \& Liem (1986) and Wada et al. (1994) to the more recent works by Fux (1999); Englmaier \& Gerhard (1999); Weiner \& Sellwood (1999) and Bissantz et al. (2003). The bar pattern rotation speed for most of those models are in the range of 40 to $60 \mathrm{~km} \mathrm{~s}^{-1} \mathrm{kpc}^{-1}$. The corotation radius is estimated to be in the range 3-4 kpc by some works (Englmaier \& Gerhard 1999; Bissantz et al. 2003; Habing et al. 2006) and in the range 4-5 kpc by other works (Combes 1996; Sevenster 1999; Fux 1999). In contrast, Binney et al. (1991) placed the corotation at $2.4 \mathrm{kpc}$ and found a pattern speed of $80 \mathrm{~km} \mathrm{~s}^{-1} \mathrm{kpc}^{-1}$. As pointed out by Combes (1996), the discrepancy between the Binney et al. estimation and other estimations can be due to the fact that the molecular gas distribution in the Galactic center region used by Binney et al. could be the response to a small nuclear bar instead of the large bar of the Galaxy.

Indeed, there is recent evidence of the presence of a distinct structure in the nuclear region of the Galaxy. The star counts map made by Alard (2001) with 2MASS data shows an excess of counts in the inner four degrees of the Galaxy that resembles a small nuclear bar. This bar could be lopsided with the barycenter displaced to negative longitudes. Nishiyama et al. (2005) have also found evidence of a structure different to the large bar or bulge in the inner degrees of the Galaxy.

In the present paper, we investigate the gas response to the mass distribution inferred from the 2MASS data. In particular, we model the central mass distribution as a small nuclear bar. We are interested in the role of this nuclear bar in explaining the differences between the results of previous works (Binney et al. 1991; Fux 1999; Combes 1996). We also want to study whether a lopsided nuclear bar could explain the apparent lopsidedness of the molecular gas distribution in the nuclear region, whose complex dynamics are far from being understood. Therefore, we have modeled the 2MASS star counts maps of Alard fitting different parametric functions using three components: a disc, bulge/bar and nuclear bar. We use that mass distribution to determine the galactic stellar potential and to model the gas dynamics using a $N$-body code that takes into account cloud collisions.

Nested bars are common in external galaxies. As much as one third of barred galaxies have a secondary nuclear bar (Laine et al. 2002). In some cases the secondary bar is coupled with the primary bar (Shaw et al. 1993) while in other cases the two bars rotate with different speeds (Wozniak et al. 1995). The present paper is the first one dealing with the gas flow in the Milky Way nested bars and we will assume that both bars are dynamically coupled (they rotate with the same speed). As we discuss in Sect. 8, this seems a good assumption since our simulations explain for the first time some characteristics of the inner Galaxy.

The paper is organized as follows: in the next section we summarize our understanding of the distribution and kinematics of the interstellar gas. In Sects. 3 and 4 we present the 2MASS star counts model and the fits to the data. The gas dynamics simulations are described in Sect. 5, and the results are presented in Sects. 6 and 7. We discuss the nature of the nuclear bar in Sect. 8. Our conclusions are summarized in Sect. 9.

\section{The distribution and kinematics of the interstellar gas}

In this section we summarize visible structures in the longitudevelocity diagram (hereafter $l v$-diagram) of the interstellar gas. The large scale $l v$-diagram derived from observations of the CO(1-0) line by Dame et al. (2001) is shown in Fig. 1 while Fig. 2 shows the $l v$-diagram of the nuclear region derived also from CO(1-0) observations by Bally et al. (1987). We also discuss the plausible face on view of the Galaxy. The different features discussed here will be used in the following sections to compare with our simulations of the gas dynamics.

\subsection{The terminal velocity curve}

The terminal velocity curve (TVC) is not a kinematic feature in itself as many independent features contribute to this curve at different Galactic longitudes. However, we discuss it here since it has been used to constrain several dynamical models of the Milky Way (Binney et al. 1991; Weiner \& Sellwood 1999; Englmaier \& Gerhard 1999; Bissantz et al. 2003). There have been different determinations of the TVC using CO and HI data (Fich et al. 1989; Burton \& Liszt 1993; Clemens 1985; Alvarez et al. 1990). The dispersion of the TVC points derived by different authors is typically $10 \mathrm{~km} \mathrm{~s}^{-1}$ (see for instance Fig. 6 of Bissantz et al. 2003). The TVC measurements by Fich et al. (1989) and Burton \& Liszt (1993) are shown in Fig. 1 and in the figures showing the simulation results. In addition, in Fig. 1 we have indicated with boxes the Sagittarius, Scutum, 


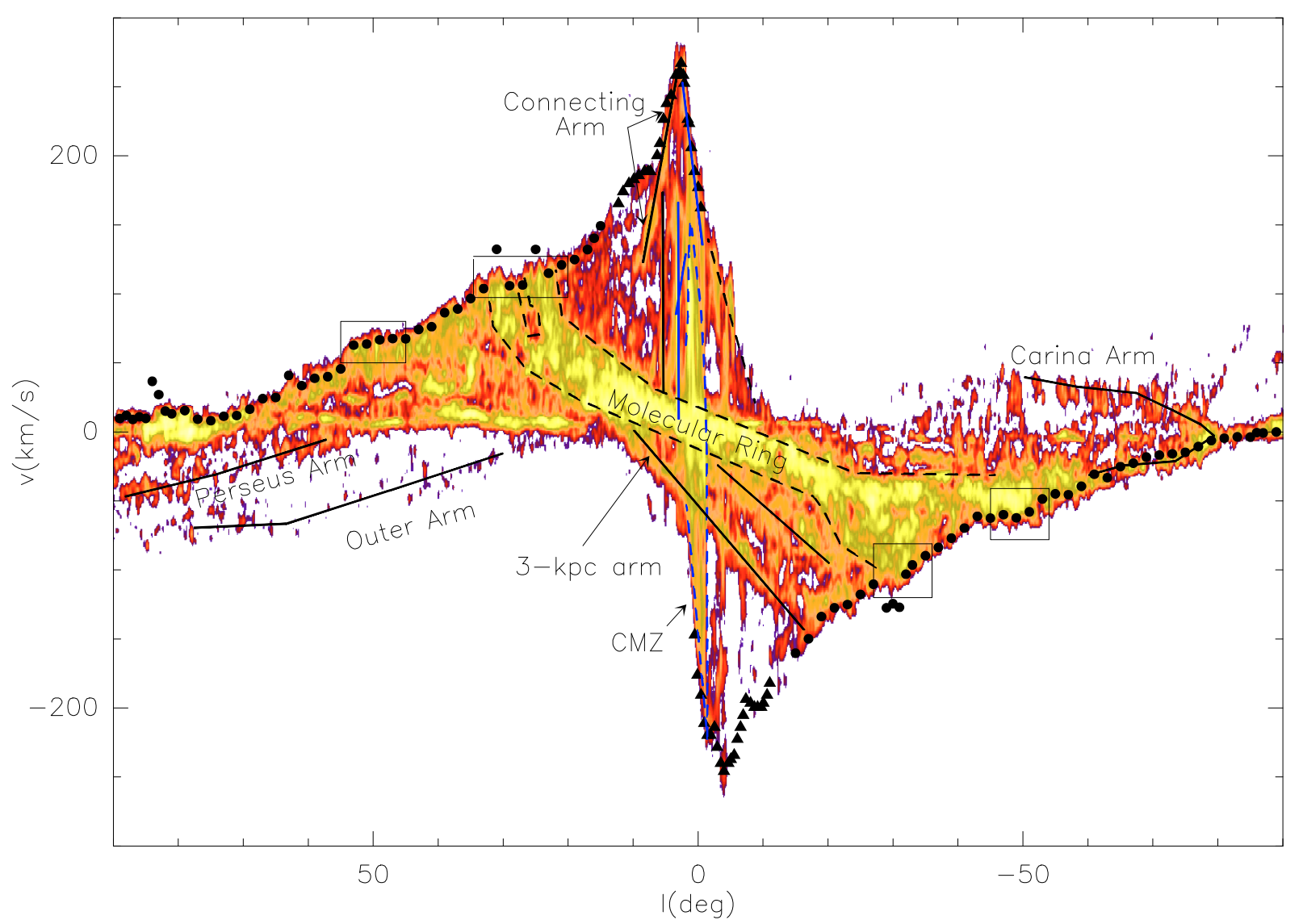

Fig. 1. Longitude-velocity $(l v)$ diagram of the $\mathrm{CO}(1-0)$ emission (Dame et al. 2001). The solid lines trace the position of some remarkable features such as the locus of the spiral arms, the 3-kpc arm and the Connecting Arm. The black dashed lines indicate the contour of the Galactic Molecular Ring. The solid circles are the terminal velocities measurements of Fich et al. (1989) using CO while the triangles are the terminal velocities determined from the HI data of Burton \& Liszt (1993). The boxes mark the position of the Sagittarius, Scutum, Norma and Centaurus tangent points, located respectively at $l \sim 50^{\circ}, 30^{\circ},-30^{\circ}$ and $-50^{\circ}$. The lines concerning the Nuclear Disk or Central Molecular Zone are blue but they are better shown in the next figure. All these lines can be used to compare with the figures showing the simulations results.

Norma and Centaurus tangent points, located respectively at $l \sim 50^{\circ}, 30^{\circ},-30^{\circ}$ and $-50^{\circ}$ (see for instance Dame et al. 2001 ; Englmaier \& Gerhard 1999).

\subsection{Spiral arms}

Many works have studied the spiral structure of the Galaxy using different tracers such as HII regions, atomic and molecular gas or photometric observations in different bands from the near to the far infrared. The different models of the spiral arms have two arms (Bash 1981, using HII regions; Drimmel \& Spergel 2001, using $K$-band observations; Benjamin 2008, using Spitzer), three (Nakanishi \& Sofue 2003; Kulkarni et al. 1982, using HI) or four arms (Georgelin \& Georgelin (1976) using HI regions; Nakanishi \& Sofue (2006) using CO or Drimmel (2000) using the 240 micron luminosity). Deprojecting a given data set to find the face on view of the Galaxy is a difficult task in particular for the inner Galaxy. For instance, the kinematical distances based in the rotation curve are not accurate due to the presence of important non-circular motion. Furthermore, after performing the deprojection there is an uncertainty as to how different structures are associated with a common arm (see Nakanishi \& Sofue 2006). Different authors have even fitted different spirals to the same data. A plausible explanation for the discrepancy between the two arms derived from stellar tracers ( $K$ band) and the four arms that are found in most of the interstellar tracers is that the Milky Way has two stellar spiral arms but the gas response is a four-armed spiral (Drimmel 2000). In any case, the major arms are logarithmic with pitch angles of $10^{\circ}-15^{\circ}$. In addition, to these grand-design spirals there is a more flocculent structure giving a number of minor arms like the Local Arm, which is a small arm linked to the Perseus spiral arm. In order to compare with our simulations, in Figs. 8, 9 and 10 we have traced the sketch of the logarithmic spiral arms of Nakanishi \& Sofue (2006), which are very similar to the classical spirals of Georgelin \& Georgelin (1976). The locus of different spiral arms in the $l v$-diagram are shown in Fig. 1 . The Carina arms deserves a special mention since the Carina tangent point is clearly seen at $l \sim-80^{\circ}$.

The determinations of the speed of the spiral pattern give values in the range from 13.5 to $59 \mathrm{~km} \mathrm{~s}^{-1} \mathrm{kpc}^{-1}$ (Lin et al. 1969; Fernández et al. 2001; Bissantz et al. 2003; Martos et al. 2004; Debattista et al. 2002). Thus, the spiral pattern speed could be lower that the bar one.

\subsection{The Galactic Molecular Ring}

The so-called Galactic Molecular Ring (GMR) is one of the most prominent structures in the $l v$-diagram (Fig. 1). The locus of the GMR is more or less a diagonal line that passes trough the origin as a circular ring would do. If the GMR is a real ring, it could be associated with a resonance. Binney et al. (1991) proposed 
that the GMR could be located at the Outer Linblad Resonance. Other models that place the corotation farther out have suggested that the GMR could be better explained by the Ultra Harmonic Resonance (Combes 1996).

However, the real spatial distribution of the GMR is not known and it could be composed of imbricated spiral arms instead of being an actual ring. Some authors (see for instance Englmaier \& Gerhard 1999) consider that, if formed by several arms, the GMR should be located outside of the corotation since they exhibit almost circular velocities. In their face-on deprojection of the neutral and molecular gas distribution, Nakanishi \& Sofue (2006) found that the GMR seems to be the inner part of the Sgr-Carina arm (the far-side of the GMR) and the ScutumCrux arm (the near-side of the GMR).

\subsection{Inner arms: the 3-kpc arm}

The 3-kpc arm is clearly seen in the $l v$-diagram of Fig. 1 with a velocity of $-53 \mathrm{~km} \mathrm{~s}^{-1}$ at $l=0^{\circ}$. The name of this feature comes from a tangent point at $l=-22^{\circ}$ that corresponds to a distance of $\sim 3 \mathrm{kpc}$. This arm is located in between the Sun and the GC since the clouds in the arm absorb the radiation from the continuum sources in the GC. The 3-kpc arm cannot be explained with a combination of rotation and expansion (Burke \& Tuve 1964) and has been interpreted as a stationary density wave in a barred potential (Mulder \& Liem 1986). The simulations of Fux (1999) suggest that it is a lateral arm that surrounds the bar while, in the simulations by Englmaier \& Gerhard (1999), it would be a small arm arising from the extremity of the bar.

Sevenster (1999) and Habing et al. (2006) propose a different explanation. Sevenster (1999) found old stars associated with the 3-kpc arm. They interpret the fact that both old stars and gas follow the same trajectories as the probe as that the arm would be the locus of closed orbits and not a spiral density wave maximum. They propose that the $3-\mathrm{kpc}$ arm has its origin near one of the two points where the bar meets its corotation radius and that the arm can be a channel to transport gas from the corotation to the GC and to fuel the star formation in cloud complexes like Sgr B2.

The recent discovery of the far-side counterpart of the 3-kpc arm by Dame \& Thaddeus (2008) will certainly contribute to our understanding of the exact nature of these features (see Sect. 6.3).

\subsection{The connecting arm and Bania's clumps: tracing the dust lanes?}

The "Connecting Arm" is clearly seen in Fig. 1 as a feature with high inclination with a velocity of $\sim 100 \mathrm{~km} \mathrm{~s}^{-1}$ at $l=10^{\circ}$ and more than $250 \mathrm{~km} \mathrm{~s}^{-1}$ at $l=3^{\circ}$. The name "Connecting Arm" comes from the fact that it seems to be connected to the nuclear ring. This is a very puzzling structure that has not been discussed much in the literature (see Fux 1999, for a short summary). On the one hand, Burton \& Liszt (1978) and Liszt \& Burton (1980) suggested that the Connecting Arm could be the edge of the HI ring found at a radius of $\sim 1 \mathrm{kpc}$ (see below). On the other hand, in one of his simulations, Fux (1999) obtained a feature in the $l v$-diagram that resembles the Connecting Arm. In that simulation, the Connecting Arm-like feature is the locus of the off-axis shocks or dustlanes (since in the optical images of barred galaxies the off-axis shocks are seen as dust lanes). Marshall et al. (2008) also interpret the Connecting Arm as the near side dustlane.

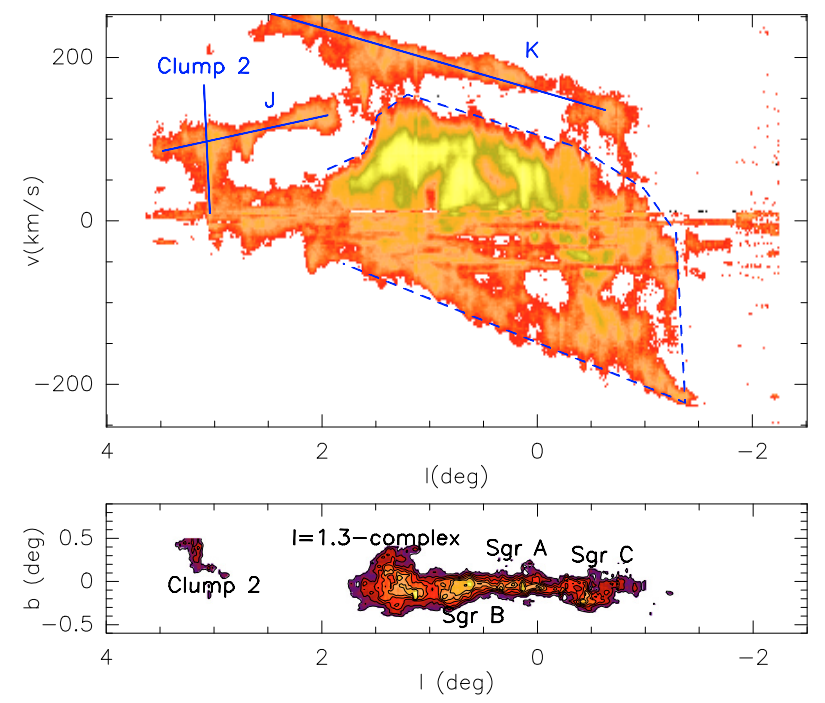

Fig. 2. Upper panel: longitude-velocity (lv) diagram of the $\mathrm{CO}(1-0)$ emission in the Central Molecular Zone using data of Bally et al. (1987) and integrating all negative Galactic latitudes. The solid lines trace the position of some remarkable features such as Clump 2, or structures K and $\mathbf{J}$ of Rodriguez-Fernandez et al. (2006). The dashed line indicates the contour of the very crowded central region. Lower panel: integrated intensity map.

However, in another of the simulations discussed by Fux (1999) the locus of the dustlane in the $l v$-diagram is a vertical feature extending approximately from a velocity of $0 \mathrm{~km} \mathrm{~s}^{-1}$ to $200 \mathrm{~km} \mathrm{~s}^{-1}$ at an almost constant longitude (see his Figs. 15 and 16). This looks like the "clumps" discussed by Bania (1977). These clumps are cloud complexes found at $l=5.5^{\circ}$ and $l=3.1^{\circ}$ that exhibit a huge velocity dispersion of $\sim 200 \mathrm{~km} \mathrm{~s}^{-1}$ in a very narrow range of longitude. A portion of the clump located at $l=3.1^{\circ}$ (known as Clump 2) is shown in Fig. 2. The Clump 2 has been interpreted as a dustlane by Stark \& Bania (1986), while in the Fux (1999) interpretation, Banias's clumps would be gas clouds that are about to enter the dustlane shocks, although the actual dustlane would be the Connecting Arm as mentioned above. This is also the interpretation of Liszt (2006, 2008), who have studied in detail the spatial and velocity structure of all these features.

\subsection{The HI ring}

The HI emission in the GC region can be modeled as a tilted circular ring with an inner radius of $\sim 300 \mathrm{pc}$ and outer radius of $1-1.5 \mathrm{kpc}$ (Burton \& Liszt 1978). This first model was improved by Liszt \& Burton (1980), who discussed that an elongated ring could also explain the observations. The spatial distribution of the interstellar gas in the innermost $3 \mathrm{kpc}$ of the Galaxy has recently been reviewed by Ferrière et al. (2007). The model that they consider more plausible, despite its inherent uncertainties, is that of Liszt \& Burton (1980) since an elongated ring seems to be easier to understand if it is composed of gas clouds moving in elongated $X_{1}$ orbits. Therefore, they modeled the HI ring as an elliptical ring with a semi-major axis of $1.6 \mathrm{kpc}$, and an axis ratio of 3.1 , with an inner hole with semi-axis of $800 \mathrm{pc} \times 258 \mathrm{pc}$ that encloses the Central Molecular Zone (see below).

\subsection{The Central Molecular Zone}

The Central Molecular Zone (hereafter CMZ) refers to the central accumulation of gas in the inner hundreds of parsec of the 


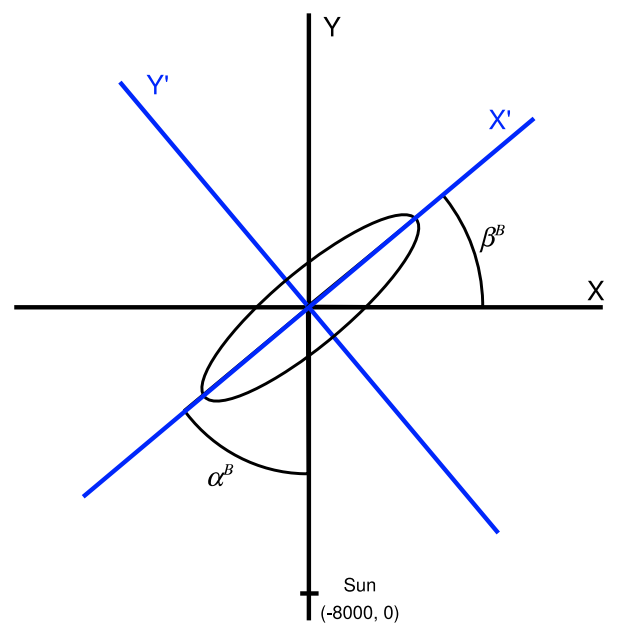

Fig. 3. Sketch of the different coordinates systems and angles used to define the bars in the Galactic plane. The $z$ and $z^{\prime}$ axis are coincident and the $z>0$ semiaxis is in the northern Galactic hemisphere.

Galaxy. This gas is mainly molecular and extends continuously from approximately $-1.5^{\circ}$ to $2^{\circ}$ (see Fig. 2). The spatial distribution is not symmetric due to the prominent cloud complex located at $l=1.3^{\circ}$ (hereafter $l=1.3^{\circ}$-complex) that lacks a negative longitude counterpart. Figure 2 also shows the $l v$ diagram of the CMZ. This plot has been obtained integrating the $\mathrm{CO}(1-0)$ data of Bally et al. (1987) for negative Galactic longitudes to reveal a number of kinematical component such as Clump 2 (at $l=3.1^{\circ}$ ), and the features $\mathrm{K}$ and $\mathrm{J}$ discussed by Rodriguez-Fernandez et al. (2006).

The kinematics of the inner region are very complex with a high fraction of the gas exhibiting non-circular velocities. Figure 2 shows with a dashed line the contour of the inner $\mathrm{CMZ}$ that contains the feature M of Rodriguez-Fernandez et al. (2006) and the inner arms found by Sofue (1995). This contour resembles a parallelogram but it is not the parallelogram that inspired the Binney et al. (1991) work.

The whole $l v$-diagram shown in Fig. 2 is very different to that used by Binney et al. (1991). These authors integrated the CO data of Bally et al. (1987) over positive and negative latitudes and studied the region $2.2^{\circ}>l>-2.2^{\circ}$. In their Fig. 2, the feature $\mathrm{K}$ is not clearly detached from the inner $l v$ parallelogram and they considered that it is part of a larger $l v$ parallelogram. However, following the analysis of the Bally et al. (1987) data by Rodriguez-Fernandez et al. (2006), we conclude that feature K is a distinct kinematic feature. This conclusion is also supported by the large scale $\left(13^{\circ}>l>-12^{\circ}\right) \mathrm{CO}(1-0)$ survey by Bitran et al. (1997), whose $l v$-diagram shows clearly that the feature $\mathrm{K}$ extends to $l \sim 3^{\circ}$ where it is connected to the Connecting Arm.

Interestingly, when one compares the $l v$ parallelograms of the $X_{1}$ orbits displayed in Fig. 3 of Binney et al. (1991) to the parallelogram shown in our Fig. 2, the upper right vertex is in good agreement, showing velocities of $\sim 0 \mathrm{~km} \mathrm{~s}^{-1}$. Thus, one of the apparent discrepancies (see Sect. 1) between the Binney et al. (1991) model and the CO lv-diagram disappears.

\section{Modeling the star count map}

\subsection{The star count map}

We have modeled the 2MASS star count map of Alard (2001) to determine a realistic stellar potential. In this section we summarize the methods and the main conclusions of Alard's work.
Using the $H$ and $K$ bands and assuming that we know the intrinsic color of the sources $(H$ and $K)$, one can correct for extinction one of the bands as follows:

$m_{K}^{\text {corr }}=m_{K}-\left[A_{K} /\left(A_{H}-A_{k}\right)\right]\left[\left(m_{H}-m_{K}\right)-(H-K)\right]$

where $m_{K, H}$ are the observed apparent magnitudes in $K$ and $H$ band (thus suffering extinction), $A_{K, H}$ is the extinction in each band and $m_{K}^{\text {corr }}$ is the extinction corrected magnitude in $K$ band. At the distance of the bulge a cutoff $m_{K}=9$ (see below) implies that the sources are early M-giants (see Lopez-Corredoira et al. 2001). Assuming an intrinsic color $H-K$ for M giants of $\sim 0.17$ (Binney \& Merrifield 1998; Wainscoat et al. 1992) and taking into account the relative extinctions $A_{k} / A_{v}=0.112$ and $A_{h} / A_{v}=0.175$ (Rieke \& Lebofsky 1985), one can write:

$m_{K}^{\text {corr }}=m_{K}-1.77\left(m_{H}-m_{K}\right)+0.30$.

Alard (2001) defined the extinction corrected magnitude $m_{\mathrm{e}} \equiv$ $m_{K}^{\text {corr }}-0.30$ as

$m_{\mathrm{e}} \equiv m_{K}-1.77\left(m_{H}-m_{K}\right)$

and constructed his star count map using this magnitude with a cutoff of 9 mag. In most of this map, the density profile at constant longitude is exponential. This exponential profile is also present in numerical simulations. Combes et al. (1990) showed that a disk with a small bulge in its center can evolve into a peanut shaped bar with a nearly exponential profile perpendicular to the plane of the galaxy. However, Alard (2001) noticed that the observed density profile in the inner four degrees of the Galaxy shows an excess of sources in the plane. Subtracting an exponential profile corresponding to disk and bulge, he found that the residual seems to be a small, lopsided, nuclear bar in the galactic center. This structure has not been found in previous studies since the data lacked both the depth and the resolution.

\subsection{The model}

In this section we describe how we have modeled the 2MASS star count map. The number of stars in a volume $\mathrm{d} V$ located at $x$ with a magnitude in the interval $M$ and $M+\mathrm{d} M$ is:

$\mathrm{d} N=\Phi(M, x) \mathrm{d} M \mathrm{~d} V$

If the distribution of magnitudes is the same in all the space we can write $\Phi$ as $\Phi(M, x)=\phi(M) \rho(x)$, where $\phi(M)$ is the luminosity function (the fraction of stars with magnitude $M$ ) and $\rho(x)$ is the star density at the point $x$. Therefore, the total number of stars with a magnitude $M$ lower than a cutoff $M_{\mathrm{c}}$ is:

$N=\int_{M<M_{\mathrm{c}}} \int_{v} \mathrm{~d} N=\int_{-\infty}^{M_{\mathrm{c}}} \int_{\mathrm{V}} \phi(M) \mathrm{d} M \rho(x, y, z) \mathrm{d} V$.

As the $K$ band luminosity function $\phi(M)$, we have used the function given by Wainscoat et al. (1992), which is very similar to that of Eaton et al. (1984). However, we work with extinctioncorrected apparent magnitudes $m$, which are related to the intrinsic magnitudes by:

$M=m+5-5 \log _{10} s$

where $s$ is the distance in pc. Therefore we actually deal with a function of the apparent magnitude and the distance $\phi^{\prime}(m, s)$.

Our model of the density distribution is given by three components: a triaxial bulge or large bar, an exponential disk and a small nuclear bar. The exact functional form of each component is given below. 


\subsubsection{Disc}

We use the exponential disc of Wainscoat et al. (1992), which is defined as:

$\rho^{D}(r, z)=\rho_{0} \exp \left(-\frac{(r-D)}{h_{\mathrm{r}}}-\frac{|z|}{h_{z}}\right)$

where $\rho_{0}$ is the star number density in the solar neighborhood, $D$ is the distance from the sun to Galactic center, $h_{z}$ and $h_{\mathrm{r}}$ are vertical and radial scale parameters, respectively.

\subsubsection{Triaxial bulge}

From the COBE/DIRBE images we know that the bulge of the Milky Way is boxy (Dwek et al. 1995). The 2MASS data confirm this result (López-Corredoira et al. 2005). Therefore, we have adopted a boxy Gaussian (Dwek et al. function G2) to represent the bulge:

$$
\begin{aligned}
\rho^{B}\left(x^{\prime}, y^{\prime}, z^{\prime}\right) & =\rho_{0} \exp \left(-0.5 r_{s}^{2}\right) \\
r_{s} & =\left(\left[\left(\frac{x^{\prime}}{x_{0}}\right)^{2}+\left(\frac{y^{\prime}}{y_{0}}\right)^{2}\right]^{2}+\left(\frac{z}{z_{0}^{\prime}}\right)^{4}\right)^{1 / 4} .
\end{aligned}
$$

The bulge function is expressed in a coordinate system $\left(x^{\prime}, y^{\prime}, z^{\prime}\right)$ that follows its symmetry axes. Therefore, a rotation is needed to obtain the expression in the $(x, y, z)$ coordinate system.

$$
\left\{\begin{array}{l}
x^{\prime}=x \cos \beta^{B}+y \sin \beta^{B} \\
y^{\prime}=-x \sin \beta^{B}+y \cos \beta^{B} \\
z^{\prime}=z .
\end{array}\right.
$$

Figure 3 shows the $(x, y)$ and $\left(x^{\prime}, y^{\prime}\right)$ axis seen from the $z=z^{\prime}>0$ hemisphere. The figure also shows the definition of the angle $\beta^{B}$, which is measured counterclockwise from the $x>0$ semi-axis. However, many papers measure the inclination of the bar clockwise from the Sun-GC line to the near side of the bar. Therefore, for an easy comparison with previous results we have defined the angle $\alpha^{B}$ as shown in the figure.

\subsubsection{Triaxial nuclear bar}

In contrast to the bulge, the small nuclear bar found by Alard does not seem to be boxy. Therefore we have taken a triaxial Gaussian function (Dwek et al. function G1) to represent this component:

$$
\begin{aligned}
\rho^{b}\left(x^{\prime \prime}, y^{\prime \prime}, z^{\prime \prime}\right) & =\rho_{0} \exp \left(-0.5 r^{2}\right) \\
r & =\left[\left(\frac{x^{\prime \prime}-x_{1}}{x_{0}}\right)^{2}+\left(\frac{y^{\prime \prime}}{y_{0}}\right)^{2}+\left(\frac{z^{\prime \prime}}{z_{0}}\right)^{2}\right]^{1 / 2}
\end{aligned}
$$

where $x_{1}$ allows us to fit a lopsided bar. To obtain the bar density in the $(x, y, z)$ coordinate system one should apply the rotation:

$$
\left\{\begin{array}{l}
x^{\prime \prime}=x \cos \beta^{b}+y \sin \beta^{b} \\
y^{\prime \prime}=-x \sin \beta^{b}+y \cos \beta^{b} \\
z^{\prime \prime}=z
\end{array}\right.
$$

The definition of $\beta^{b}$ is exactly analogous to that of $\beta^{B}$ shown in Fig. 3. Again, in the following, and for coherence with most of the literature in the subject, we will use an angle $\alpha^{b}$ measured clockwise from the Sun-GC line to refer to the inclination of the nuclear bar.

\subsubsection{Final star counts equation}

Finally, we must also convert $\rho(x, y, z)$ to $\rho^{\prime}(l, b, s)$ since we want to model the star counts in Galactic coordinates $(l, b)$ and to integrate along the line of sight $(s)$. If $(x, y, z)$ is a right-handed reference frame centered in the galactic center, with the plane of the sky in the $x z$-plane with $x>0$ for $l<0$, and $z>0$ towards the Galactic north pole, and assuming that the sun is located at $(x, y, z)=(0,-D, 0)$ then:

$$
\begin{aligned}
& x=-s \cos (b) \sin (l) \\
& y=-D+s \cos (b) \cos (l) \\
& z=s \sin (b) .
\end{aligned}
$$

The star counts equation towards $\left(l_{0}, b_{0}\right)$ is then:

$$
\begin{aligned}
N\left(l_{0}, b_{0}, m_{\mathrm{c}}\right) & =\Delta l \Delta b \\
& \times \int_{m_{\min }}^{m_{\max }} \mathrm{d} m \int_{0}^{s_{\max }} \mathrm{d} s \phi^{\prime}(m, s) \rho^{\prime}\left(l_{0}, b_{0}, s\right) s^{2} \cos b
\end{aligned}
$$

where $s^{2} \cos b$ is the Jacobian of the coordinate transformation (Eq. (14)). We have assumed that the density is constant in the interval from $l_{0}$ to $l_{0}+\Delta l$ and from $b_{0}$ to $b_{0}+\Delta b$.

We have computed the integral in magnitudes from $m_{\min }=$ $-10.0 \mathrm{mag}$ to $m_{\max }=9.0 \mathrm{mag}$ with $\Delta m=0.5 \mathrm{mag}$. The integral of the distance along the line of sight $s$ has been computed up to $s_{\max }=20 \mathrm{kpc}$ using a variable step $\Delta s$ :

$$
\Delta s= \begin{cases}0.002 \mathrm{kpc} & s, R<0.100 \mathrm{kpc} \\ 0.008 \mathrm{kpc} & s, R<0.300 \mathrm{kpc} \\ 0.010 \mathrm{kpc} & s, R<0.700 \mathrm{kpc} \\ 0.050 \mathrm{kpc} & s, R<1.500 \mathrm{kpc} \\ 0.100 \mathrm{kpc} & s, R<3.100 \mathrm{kpc} \\ 0.200 \mathrm{kpc} & s, R<6.300 \mathrm{kpc} \\ 0.300 \mathrm{kpc} & s, R>6.300 \mathrm{kpc} .\end{cases}
$$

\section{Fitting the star count map}

We have fitted the star count map using the model described above. This model has 14 free parameters corresponding to the disc $\left(\rho_{0}, h_{\mathrm{r}}, h_{z}\right)$, the bulge $\left(\rho_{0}, x_{0}, y_{0}, z_{0}, \alpha\right)$ and the nuclear bar $\left(\rho_{0}, x_{0}, y_{0}, z_{0}, \alpha, x_{1}\right)$.

The star count equation is evaluated for an initial guess of the density function parameters. A $\chi^{2}$ parameter is computed as the difference of the star count data points $\left(N_{\text {data }_{i}}\right)$ and the model $\left(N_{\text {model }_{i}}\right)$,

$\chi^{2}=\frac{1}{n} \sum_{i=1}^{n} \frac{\left(N_{\mathrm{data}_{i}}-N_{\text {model }_{i}}\right)^{2}}{\sigma_{i}^{2}}$

where $n$ is the number of points to be fitted and $\sigma$ is the error of the data points. We have estimated the $\sigma$ from Fig. 1 of Alard (2001) as the dispersion of the star counts with respect to an exponential vertical profile. Afterwards we used an iterative process to minimize $\chi^{2}$ using the Levenberg-Marquardt algorithm (Press et al. 1992).

\subsection{Fitting the disc and the boxy bulge}

We have not attempted to fit all the free parameters at once. First, we fitted a star count model with the disc and the boxy bulge to a reduced data set consisting of one point every 10 pixels (one data point every $0.5^{\circ}$ ). In the disc region, we took one point every 5 pixels $\left(0.25^{\circ}\right)$ across the disc. Finally, we neglected the data points in the inner region $(-3<l(\mathrm{deg})<3,-2<b(\mathrm{deg})<2$ ) since they are clearly dominated by the nuclear bar. This reduced 


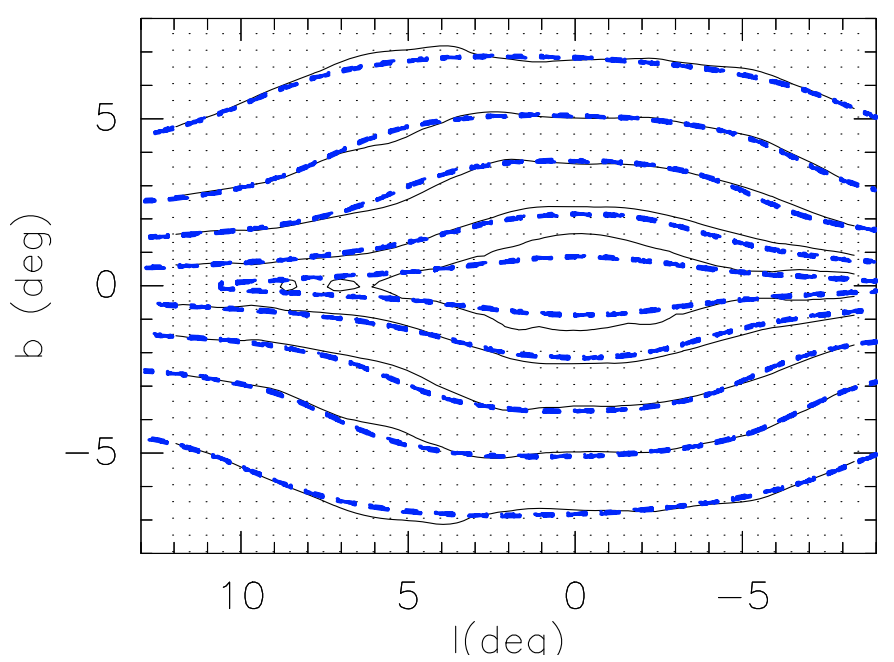

Fig. 4. Thin solid contours represent the star counts map. Thick dashed lines are the best fit obtained with a star count model with $\alpha^{B}=20^{\circ}$ and $h_{\mathrm{r}}=2.5 \mathrm{kpc}$. The points show the data set used to fit the disc and bulge.

data set contains 1494 points and is shown in Fig. 4. The error of these data points used to calculate $\chi^{2}$ was estimated to be $15 \%$ as explained above.

We have fitted the data for fixed values of the bulge angle $\left(\alpha^{B}=60,45,35,20,10,0 \mathrm{deg}\right)$ and the disc radial scale $\left(h_{\mathrm{r}}=1.5,2,2.5,3.5 \mathrm{kpc}\right)$. The results obtained for the other free parameters are shown in Table 1. For all $\alpha^{B}$, the best fits are always obtained with $h_{\mathrm{r}}=2.5 \mathrm{kpc}$. This is in agreement with the radial scale length of the disc derived from previous studies in the infrared, which have values in the range from 1.9 (2MASS López-Corredoira et al. 2005) to 2.6 (COBE Freudenreich 1998). Regarding the angle of the bulge, the best fit is obtained for $10^{\circ}$, although $\chi^{2}$ is only $2.8 \%$ higher for $\alpha^{B}=20^{\circ}$ (Fig. 4 shows the best fit with $\alpha^{B}=20^{\circ}$ ). This is also in agreement with other studies of the structure of the inner Galaxy. Dwek et al. (1995) derived angles in the range $16^{\circ}-$ $40^{\circ}$ depending on the function assumed to represent the bulge (they found $16^{\circ}$ with the same boxy Gaussian that we use here), while Freudenreich (1998) derived angles in the range of $9^{\circ}-$ $15^{\circ}$ also using COBE/DIRBE data. A high angle of $12^{\circ}$ has also been measured from the 2 Micron Galaxy Survey (TMGS) by López-Corredoira et al. (2000). On the other hand, the work of López-Corredoira et al. (2005) using 2MASS data found $\alpha^{B}=$ $20^{\circ}-35^{\circ}$.

The shape of the bulge as given by the best fit with $\alpha^{B}=10^{\circ}$ is $x_{0} / x_{0}: y_{0} / x_{0}: z_{0} / x_{0}=1: 0.5: 0.3$ while the best fit with $\alpha^{B}=$ $20^{\circ}$ has axis ratios of 1:0.55:0.4 which are in perfect agreement with those derived by López-Corredoira et al. (2005), also with 2MASS data or by López-Corredoira et al. (2000) with TMGS data. In contrast, other studies have favored somewhat thinner bulges with ratios of 1:0.3-0.4:0.3 (Dwek et al. 1995; Freudenreich 1998; Bissantz et al. 2003).

\subsection{Fitting disc, bulge and nuclear bar}

In the second data set we have also included the star counts in the innermost region of the Galaxy $(-3<l<3,-2<b<2)$. We have taken one point every 3 pixels $\left(0.15^{\circ}\right)$ across the disc. The data set is shown in Fig. 5. It contains 1734 points. From Fig. 1 of Alard (2001) it is clear that the dispersion of the star count map in the nuclear region is narrower than in the bulge. We have estimated a $\sigma$ of $5 \%$ for these data points.

We have fitted the data using our star count model with three components: disc, bulge and nuclear bar. We have taken as a starting point the best fits for $\alpha^{B}=10^{\circ}, 20^{\circ}, 35^{\circ}, 45^{\circ}$ and $60^{\circ}$ derived in the previous section (all the parameters of the disc and the bulge are fixed except the factors $\rho_{0}$ ). The best fit results for the parameters of the nuclear bar are summarized in Table 2. The full set of results for the nuclear bar fits are listed in Tables 3-6.

The best fits are found with a thick triaxial bar with typical axis ratios of $1:(0.7-0.8):(0.5-0.6)$ and $\alpha^{b}$ in the interval 60-120 for all the $\alpha^{B}$. This implies that the parameters of the nuclear bar can indeed be constrained independently of the bulge and that $\alpha^{b} \neq \alpha^{B}$. Globally, the best fit is obtained with $\alpha^{B}=10^{\circ}$ and a nuclear bar with $\alpha^{b}=90^{\circ}-105^{\circ}$. Some examples of good fits are shown in Fig. 5.

Finally, we have also performed fits with a lopsided nuclear bar $\left(x_{1} \neq 0\right)$. The results are shown in Tables 3-6 and they are summarized in Table 2. The best fits are still obtained with a nuclear bar that is almost perpendicular to the Sun-GC line. The symmetry center of the bar would be shifted by $22-25$ pc towards the third Galactic quadrant if $\alpha^{b}=60^{\circ}-90^{\circ}$ while it would be shifted by the same quantity but towards the fourth quadrant if $\alpha^{b}=90^{\circ}-120^{\circ}$.

\section{Simulations of the gas dynamics: methods}

We have used the code of Combes \& Gerin (1985) to simulate the motion of gas clouds in the potential obtained from the mass distribution inferred with the star count model. This code has already been used successfully to understand the gas distribution and dynamics of a number of galaxies such as M100 (Garcia-Burillo et al. 1998), or NGC 4736 (Gerin et al. 1991; Mulder \& Combes 1996).

\subsection{Stellar potential, rotation curve and masses}

The potential is obtained from the mass distribution derived in the previous section, using standard FFT techniques in a Cartesian grid of $512 \times 512 \times 16$ cells. The cells are cubic with a linear size of $58.6 \mathrm{pc}$, therefore the physical size of the grid is $30 \times 30 \times 0.94 \mathrm{kpc}^{3}$. This is good compromise to model the large scale dynamics at least to a radius of $10 \mathrm{kpc}$ and to have a good enough spatial resolution to study the Galactic center.

As described by Combes \& Sanders (1981), the interaction between periodically reproduced images is avoided following the scheme of Hohl \& Hockney (1969), i.e. the potential computations are done in a grid 8 times bigger, of $1024 \times 1024 \times$ 32 cells. The disk mass is truncated at $15 \mathrm{kpc}$ in radius, and the mass distribution is assumed spherical outside, so that its influence in the center is cancelled. This is equivalent to assuming that a spherical dark halo dominates the mass outside. This assumption should have a negligible influence on the dynamics of the inner Galaxy, which is the main interest of this paper.

The stellar potential is decomposed into its axisymmetric and non-axisymmetric parts. The axisymmetric potential is calculated in radial bins using the averaged mass in the bin. The nonaxisymmetric part of the potential is calculated with a mass distribution corresponding to the difference between the total mass and the axisymmetric distribution.

The stellar number density derived in the previous section should be multiplied by a factor $f$ to obtain the total mass density. This factor accounts for the mass of the stars detected by 
Table 1. Results of the fits to the star counts data of Fig. 4 using an exponential disc and a triaxial boxy-Gaussian bulge.

\begin{tabular}{lllllllll}
\hline \hline & \multicolumn{4}{c}{ Bulge } & \multicolumn{5}{c}{ Disk } \\
$x_{0}$ & $y_{0}$ & $z_{0}$ & $\rho_{0}$ & $\alpha^{B}$ & $h_{\mathrm{r}}$ & $h_{z}$ & $\rho_{0}$ & $\chi^{2}$ \\
$\mathrm{kpc}$ & $\mathrm{kpc}$ & $\mathrm{kpc}$ & & $\mathrm{deg}$ & $\mathrm{kpc}$ & $\mathrm{kpc}$ & & \\
\hline 1.068 & 0.498 & 0.346 & $0.389 \mathrm{E}-02$ & 0.0 & 2.500 & 0.168 & $0.249 \mathrm{E}-03$ & 0.412 \\
1.117 & 0.513 & 0.362 & $0.383 \mathrm{E}-02$ & 10.0 & 2.500 & 0.153 & $0.254 \mathrm{E}-03$ & 0.259 \\
1.243 & 0.540 & 0.383 & $0.303 \mathrm{E}-02$ & 10.0 & 2.000 & 0.161 & $0.145 \mathrm{E}-03$ & 0.264 \\
0.817 & 0.491 & 0.349 & $0.615 \mathrm{E}-02$ & 20.0 & 3.500 & 0.134 & $0.469 \mathrm{E}-03$ & 0.336 \\
0.840 & 0.486 & 0.359 & $0.557 \mathrm{E}-02$ & 20.0 & 3.000 & 0.144 & $0.366 \mathrm{E}-03$ & 0.288 \\
0.884 & 0.489 & 0.375 & $0.477 \mathrm{E}-02$ & 20.0 & 2.500 & 0.156 & $0.255 \mathrm{E}-03$ & 0.266 \\
0.975 & 0.513 & 0.400 & $0.376 \mathrm{E}-02$ & 20.0 & 2.000 & 0.166 & $0.145 \mathrm{E}-03$ & 0.284 \\
1.118 & 0.574 & 0.432 & $0.283 \mathrm{E}-02$ & 20.0 & 1.500 & 0.169 & $0.551 \mathrm{E}-04$ & 0.325 \\
0.703 & 0.444 & 0.355 & $0.768 \mathrm{E}-02$ & 35.0 & 3.500 & 0.135 & $0.470 \mathrm{E}-03$ & 0.335 \\
0.716 & 0.435 & 0.366 & $0.708 \mathrm{E}-02$ & 35.0 & 3.000 & 0.145 & $0.366 \mathrm{E}-03$ & 0.291 \\
0.744 & 0.429 & 0.382 & $0.621 \mathrm{E}-02$ & 35.0 & 2.500 & 0.158 & $0.255 \mathrm{E}-03$ & 0.274 \\
0.812 & 0.442 & 0.408 & $0.499 \mathrm{E}-02$ & 35.0 & 2.000 & 0.169 & $0.145 \mathrm{E}-03$ & 0.301 \\
0.937 & 0.496 & 0.445 & $0.371 \mathrm{E}-02$ & 35.0 & 1.500 & 0.172 & $0.551 \mathrm{E}-04$ & 0.350 \\
0.670 & 0.381 & 0.369 & $0.851 \mathrm{E}-02$ & 45.0 & 3.000 & 0.146 & $0.367 \mathrm{E}-03$ & 0.293 \\
0.693 & 0.367 & 0.385 & $0.767 \mathrm{E}-02$ & 45.0 & 2.500 & 0.159 & $0.255 \mathrm{E}-03$ & 0.278 \\
0.752 & 0.365 & 0.412 & $0.637 \mathrm{E}-02$ & 45.0 & 2.000 & 0.171 & $0.145 \mathrm{E}-03$ & 0.309 \\
0.621 & 0.200 & 0.373 & $0.171 \mathrm{E}-01$ & 60.0 & 3.000 & 0.147 & $0.367 \mathrm{E}-03$ & 0.297 \\
0.637 & 0.132 & 0.389 & $0.226 \mathrm{E}-01$ & 60.0 & 2.500 & 0.161 & $0.254 \mathrm{E}-03$ & 0.286 \\
0.681 & 0.179 & 0.416 & $0.140 \mathrm{E}-01$ & 60.0 & 2.000 & 0.172 & $0.144 \mathrm{E}-03$ & 0.327 \\
\hline
\end{tabular}
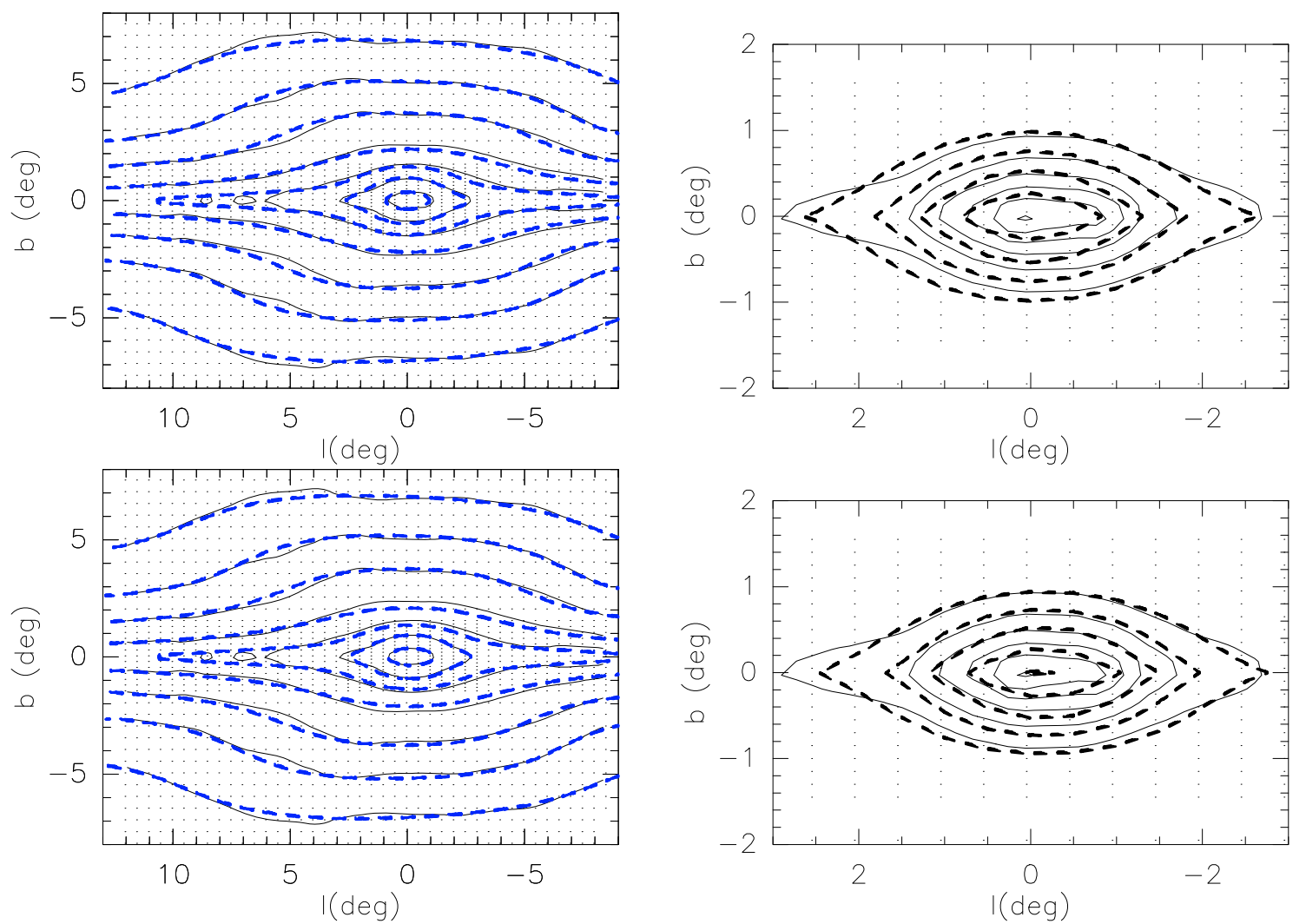

Fig. 5. Upper panels: thin solid contours represent the star count map. Thick dashed lines are the best fit obtained with a star count model with $\alpha^{B}=10^{\circ}, h_{\mathrm{r}}=2.5 \mathrm{kpc}$ and $\alpha^{b}=75 \mathrm{deg}$. The panel on the right is a zoom of the nuclear region. The points indicate the data used to do the fit. Lower panels: same that the upper panels for the best fit with $\alpha^{B}=45^{\circ}$, and a lopsided bar with $\alpha^{b}=60^{\circ}$.

2MASS but also for the mass of those stars that are not counted in the 2MASS maps. In addition, we have to introduce a dark halo to explain the observed velocities at large radii. The total density used is then:

$\rho^{T}=f\left(\rho^{D}+\rho^{B}+\rho^{b}\right)+\rho^{H}$ where the halo density is defined by a Plummer law:

$\rho^{H}(r)=\frac{\rho_{0}^{H}}{\left[1+\left(r / r_{\mathrm{c}}\right)^{2}\right]^{5 / 2}}$.

To determine the scaling factors $f$ and $\rho_{0}^{H}$ we have compared the terminal velocities and the rotation curve of the models with 
Table 2. Summary of the parameters of the nuclear bar as derived from the best fits to the 2MASS star count data.

\begin{tabular}{|c|c|c|c|c|c|c|}
\hline $\begin{array}{l}x_{0}^{b} \\
\mathrm{kpc}\end{array}$ & $\begin{array}{l}y_{0}^{b} \\
\mathrm{kpc}\end{array}$ & $\begin{array}{l}z_{0}^{b} \\
\mathrm{kpc}\end{array}$ & $\rho_{0}^{b}$ & $\begin{array}{l}\alpha^{b} \\
\operatorname{deg}\end{array}$ & $\begin{array}{l}x_{1} \\
\mathrm{kpc}\end{array}$ & $\chi^{2}$ \\
\hline $0.141-0.150$ & $0.130-0.138$ & $0.095-0.097$ & $0.141-0.150$ & $60-120$ & 0.0 & $0.357-0.376$ \\
\hline $0.158-0.166$ & $0.115-0.133$ & $0.088-0.094$ & $0.151-0.178$ & $60-120$ & $0.022-0.025$ & $0.331-0.346$ \\
\hline
\end{tabular}

The first row are fits with $x_{1}$ fixed to 0 and the second row corresponds to fits with free $x_{1}$ to allow for lopsidedness. The full set of results as a function of the orientation of the bulge $\left(\alpha^{B}\right)$ are listed in Tables 3 to 6 .

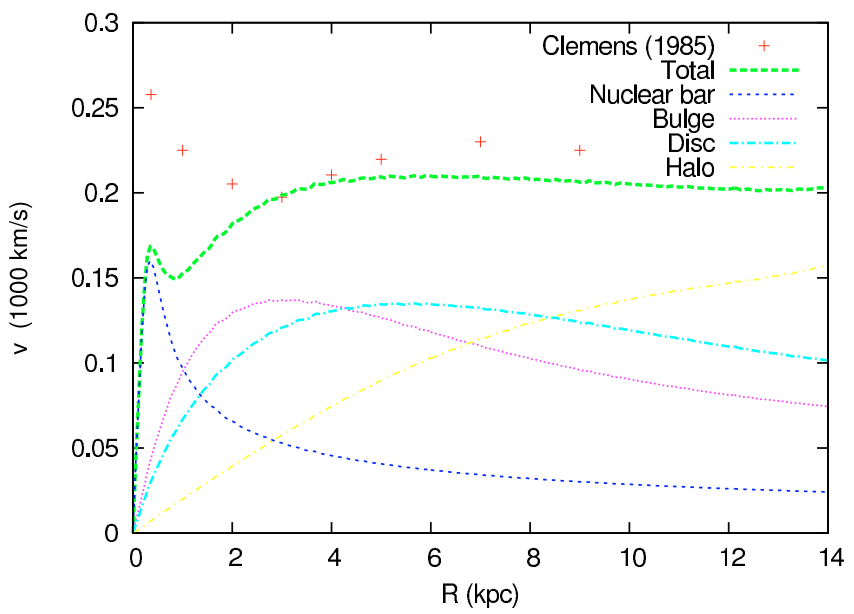

Fig. 6. Rotation curve obtained with the halo and the scaling discussed in Sect. 5.1 for a bulge and nuclear bar orientation of $\alpha^{B}=20^{\circ}$ and $\alpha^{b}=75^{\circ}$, respectively. The different lines show the contribution of the different mass components to the total rotation curve. The red points indicate the rotation curve measured from observational data. The mass of the halo, disc, and bulge are $M^{H}=7 \times 10^{10} M_{\odot}, M^{D}=2.9 \times 10^{10} M_{\odot}$, $M^{B}=1.9 \times 10^{10} M_{\odot}$, respectively. The mass of the nuclear bar is $M^{b}=$ $2.0 \times 10^{9} M_{\odot}$.

those derived from spectroscopic observations (Clemens 1985; Fich et al. 1989; Burton \& Liszt 1993). To obtain terminal velocities of the right magnitude (see Figs. 8 and 9) and a flat rotation curve at large radii (Fig. 6), one should apply a scaling factor $f=3.9 \times 10^{8}$ and a halo with $\rho_{0}^{H}=1.12 \times 10^{5}$ and $r_{\mathrm{c}}=15.0 \mathrm{kpc}$. With these factors and the parameters given in Tables $3-6$, the function $\rho^{T}$ gives the total mass density in units of $10^{3} M_{\odot} / \mathrm{kpc}^{3}$. Integrating over the total grid size, the typical masses of the different components are: $M^{H}=7 \times 10^{10} M_{\odot}, M^{D}=2.9 \times 10^{10} M_{\odot}$, $M^{B}=1.9 \times 10^{10} M_{\odot}$ and $M^{b}=2.0 \times 10^{9} M_{\odot}$, which are in good agreement with previous determinations (see for instance Weiner \& Sellwood 1999; Fux 1999).

Figure 6 shows an example of a rotation curve. The modeled curve compares well with the rotation curve derived from observations of the interstellar gas for radii larger than $2 \mathrm{kpc}$. The agreement seems worse for small radii. However the comparison in this region is not straightforward. On the one hand, the measured curve has been derived using the tangent point method assuming that the clouds move in circular orbits, which is not true in the innermost region. In addition, this curve depends on the position of the observer in the Galaxy. On the other hand, the modeled rotation curve has been derived with the axisymmetrical potential and with an azimuthal average of the nonaxisymmetrical potential. This rotation curve is an average curve independent of the position of the observer.

Figure 7 gives the rotation velocity $\Omega$ as a function of galactocentric radius. It also shows the $\Omega+\kappa / m$ curves (where $\kappa$ is the epicyclic frequency). For a given bar pattern speed $\Omega_{\mathrm{p}}$, these

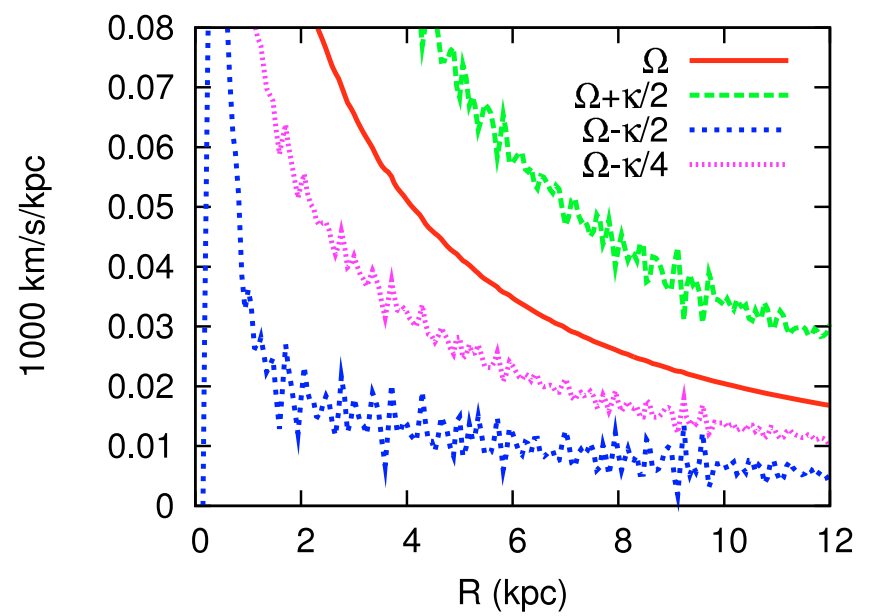

Fig. 7. Angular frequencies as a function of radius in the epicyclic approximation for the potential discussed in Sect. 5.1.

curves give the location of the corotation $\left(\Omega=\Omega_{\mathrm{p}}\right)$, the Outer Lindblad resonance $\left(\Omega+\kappa / 2=\Omega_{\mathrm{p}}\right)$, the Inner Lindblad resonances $\left(\Omega-\kappa / 2=\Omega_{\mathrm{p}}\right)$ and the Ultra Harmonic Resonance $\left(\Omega-\kappa / 4=\Omega_{\mathrm{p}}\right)$.

\subsection{Gas cloud dynamics}

The gas clouds are modeled as particles that are initially launched in the axisymmetric potential with a velocity corresponding to the circular velocity of the potential at the radius of the particle. The initial radial distribution is an exponential disk with a radial scale of $2 \mathrm{kpc}$. The non-axisymmetric part of the potential is then introduced gradually multiplying by the factor $1-\exp [-t / \tau]^{2}$ with a time delay of $\tau=100 \mathrm{Myr}$. The angular speed of the non-axisymmetrical pattern, $\Omega_{\mathrm{p}}$, is constant along the simulation.

We do not use a mass spectrum for the clouds and we do not simulate star formation. The simulations typically contain $10^{6}$ particles. The effects of gas self-gravitation are neglected. The clouds move as test particles in the stellar potential except when they collide inelastically. The forces due to the stellar potential are evaluated every $1 \mathrm{Myr}$ and the particles are moved according to them. The particles are followed in three dimensions but the collisions are computed in a two-dimensional grid with $480 \times 480$ cells. Clouds in the same grid cell can collide. The relative velocity of two collision partners loses $60 \%$ of its absolute value in the collision. A detailed discussion of the collisional schema can be found in Combes \& Gerin (1985) and Casoli \& Combes (1982). 


\section{Simulation results and discussion: large scale structure}

Figures 8 and 9 show face-on views and the $l v$-diagram for a grid of models with $\alpha^{B}$ from $10^{\circ}$ to $45^{\circ}$ and pattern speeds from 20 to $50 \mathrm{~km} \mathrm{~s}^{-1} \mathrm{kpc}^{-1}$. For an easy comparison with observations, we have overlaid in the $l v$-diagrams the TVC points, the boxes indicating the tanget points and the solid and dashed lines shown in Fig. 1. We have also plotted the four logarithmic spirals of Nakanishi \& Sofue (2006) in the face-on views. The models shown in Figs. 8 and 9 exhibit a number of common characteristics. The galaxies have two major spiral arms but they also exhibit a second pair of less prominent spiral arms. In the inner $\sim 4 \mathrm{kpc}$, there are arms that extend around the bar forming an oval (hereafter lateral arms). The density of gas inside this oval decreases to a radius of $\sim 1 \mathrm{kpc}$, where there is a ring. This ring is connected to the lateral arms by the off-axis arms (or dustlanes). In the inner hundreds of parsecs there is a nuclear condensation of gas.

The Milky Way galaxy is a very complex system and up to now there are no simulations of the gas dynamics that can explain all the observed features, neither quantitatively nor qualitatively. Englmaier \& Gerhard (1999) have compared their models to the terminal velocity curve, they have also compared the position the arm tangent points in the CO $l v$-diagram, at $|l|=30^{\circ}$ and $50^{\circ}$. The two $l v$-diagrams presented by Fux (1999) were selected by the global resemblance to the $l v$-diagram s obtained from $\mathrm{CO}$ and HI data. They reproduce qualitatively the 3-kpc arm and the Connecting Arm. Weiner \& Sellwood (1999) constrained their models by comparing them with the HI terminal velocity curve. Finally, Bissantz et al. (2003) used mainly the terminal velocity curve and some voids in the observed $l v$-diagram to compare with their simulations. To select the best models, in the present work we have compared with: i) the terminal velocity curve, ii) the inclination of the GMR in the $l v$-diagram, iii) the existence or not of the $3 \mathrm{kpc}$ arm and when it exists, its inclination and absolute velocity iv) the arm tangent points v) the shape and size of the HI $1 \mathrm{kpc}$ ring.

\subsection{Pattern speed and orientation of the bulge}

Figure 8 shows face-on views and $l v$-diagrams for a grid of models with $\alpha^{B}=20^{\circ}$ and pattern speeds from 20 to $50 \mathrm{~km} \mathrm{~s}^{-1} \mathrm{kpc}^{-1}$. The model with $\Omega_{\mathrm{p}}=20 \mathrm{~km} \mathrm{~s}^{-1} \mathrm{kpc}^{-1}$ reproduces some spiral tangent points and has an inner lateral arm with the inclination of the 3-kpc arm, however it does not reproduce the terminal velocity curve nor the velocity of the $3-\mathrm{kpc}$ arm, for instance. At the other extreme, the model with $\Omega_{\mathrm{p}}=50 \mathrm{~km} \mathrm{~s}^{-1} \mathrm{kpc}^{-1}$ does not reproduce satisfactorily either the terminal velocity curve or the GMR or the 3 -kpc arm.

In contrast, the models with $\Omega_{\mathrm{p}}=30$ and $40 \mathrm{~km} \mathrm{~s}^{-1} \mathrm{kpc}^{-1}$ give a very good overall representation of the Galaxy. Both reproduce satisfactorily the spiral tangent points at $|l|=30,50^{\circ}$ within $\pm 5^{\circ}$ and $\pm 10 \mathrm{~km} \mathrm{~s}^{-1}$, approximately. In addition, the model with $\Omega_{\mathrm{p}}=40 \mathrm{~km} \mathrm{~s}^{-1} \mathrm{kpc}^{-1}$ reproduces very well the Carina arm and its tangent point at $\approx-80^{\circ}$. However, the locus of the inner spiral arms and the $3-\mathrm{kpc}$ arm in the modeled $l v$-diagram are too steep in comparison with the observed $l v$ diagram. In contrast, the GMR and the the 3-kpc arm is very well reproduced from $|l|=90^{\circ}$ to $15^{\circ}$ by the model with $\Omega_{\mathrm{p}}=30 \mathrm{~km} \mathrm{~s}^{-1} \mathrm{kpc}^{-1}$. In particular the $3-\mathrm{kpc}$ arm is reproduced with both the correct inclination and the velocity within $\sim 10 \mathrm{~km} \mathrm{~s}^{-1}$. In addition, the terminal velocity curve for $l>5^{\circ}$ is very well reproduced. Given the difficulty of the task, it will not be realistic to give a very narrow interval for the bar pattern speed; from our best models we conclude that the most probable value for $\Omega_{\mathrm{p}}$ should be in the range $30-40 \mathrm{~km} \mathrm{~s}^{-1} \mathrm{kpc}^{-1}$. However, since this work is devoted to the inner Galaxy structure and kinematics, in the following we will mainly discuss models with $\Omega_{\mathrm{p}}=30 \mathrm{~km} \mathrm{~s}^{-1} \mathrm{kpc}^{-1}$ since they explain the terminal velocity curve, the 3-kpc arm and the GMR better than the model with $\Omega_{\mathrm{p}}=40 \mathrm{~km} \mathrm{~s}^{-1} \mathrm{kpc}^{-1}$.

Figure 9 shows face-on views and $l v$-diagrams for a grid of models with constant $\Omega=30 \mathrm{~km} \mathrm{~s}^{-1} \mathrm{kpc}^{-1}$ and $\alpha^{B}$ of $45^{\circ}$, $35^{\circ}, 20^{\circ}$ and $10^{\circ}$ ( $\alpha^{b}$ is constant to $90^{\circ}$ for all the models). For $\alpha^{B}=10^{\circ}$, the velocity along the line of sight is too low to explain the high non-circular velocities of the $3 \mathrm{kpc}$ arm $\left(-53 \mathrm{~km} \mathrm{~s}^{-1}\right.$ at $l=0^{\circ}$ ) and the observed arm is not reproduced in the $l v$ diagram. In contrast, the 3-kpc arm and the inclination of the GMR is well reproduced for $\alpha^{B}$ from $20^{\circ}$ to $45^{\circ}$. Nevertheless, the model with $\alpha^{B}=45^{\circ}$ does not correctly reproduce the positive velocity terminal curve. In addition, there is no ring at $1 \mathrm{kpc}$ in the faceon view.

Once again, we cannot give a very narrow interval of angles for the inclination angle of the bar since the analysis, as in other published works, is still rather qualitative. We reckon that the inclination of the bar should be in the range of $20^{\circ}$ to $35^{\circ}$, with some preference for $20^{\circ}$ since the quantitative agreement of the 3 -kpc arm and the terminal velocity curve is better. Therefore, the model with $\Omega_{\mathrm{p}}=30 \mathrm{~km} \mathrm{~s}^{-1} \mathrm{kpc}^{-1}$ and $\alpha^{B}=20^{\circ}$ will be considered as our standard model in the rest of the paper.

\subsubsection{Comparison with previous works}

The inclination of the bar derived from our models is in good agreement with previous determinations (Binney et al. 1997; Fux 1999; López-Corredoira et al. 2005, see also Sect. 1). Regarding the bar pattern speed, our simulations give results that are lower than those of Englmaier \& Gerhard (1999) or Bissantz et al. (2003), which suggest $\Omega_{\mathrm{p}}=50-60 \mathrm{~km} \mathrm{~s}^{-1} \mathrm{kpc}^{-1}$, but in good agreement with the results of Weiner \& Sellwood (1999), $\Omega_{\mathrm{p}}=42 \mathrm{~km} \mathrm{~s}^{-1} \mathrm{kpc}^{-1}$, or Fux (1999). In his self-consistent gas and star simulations, Fux found that the pattern speed evolves from 50 to $30 \mathrm{~km} \mathrm{~s}^{-1} \mathrm{kpc}^{-1}$. Taking into account our potential, a pattern speed of 30 or $40 \mathrm{~km} \mathrm{~s}^{-1} \mathrm{kpc}^{-1}$ gives a corotation radius of 7 and $5 \mathrm{kpc}$, respectively (see Fig. 7). Therefore, the corotation radius is well beyond the bar extremity, which is the characteristic of a slow bar (see for instance Sellwood \& Sparke 1988).

To explain the fact that the dustlanes in the inner spiral arms of some galaxies are located in the concave section of the arms, and assuming that the bars are fast (i.e., they extend to the corotation), Sellwood \& Sparke (1988) proposed the existence of different speeds for the bar and the spiral patterns. Indeed, for the Milky Way some works have derived low rotations speeds of 13-20 km s $\mathrm{kpc}^{-1}$ for the spiral pattern (Lin et al. 1969; Morgan 1990; Amaral \& Lepine 1997). Gas flow models with independent speeds for the bar and the spiral patterns have been presented by Bissantz et al. (2003), who discussed that different speeds for the spiral and the bar patterns could explain some regions devoid of gas in the $l v$-diagram. However, the overall agreement of their $l v$-diagrams with observations is not better than other models like that of Fux (1999) or our models, which are able to reproduce quantitatively features as the 3-kpc arm.

It is interesting to remark that our simulations favor a relatively slow bar and not a fast bar as assumed by Sellwood \& Sparke (1988). Furthermore, the pattern speed of the 

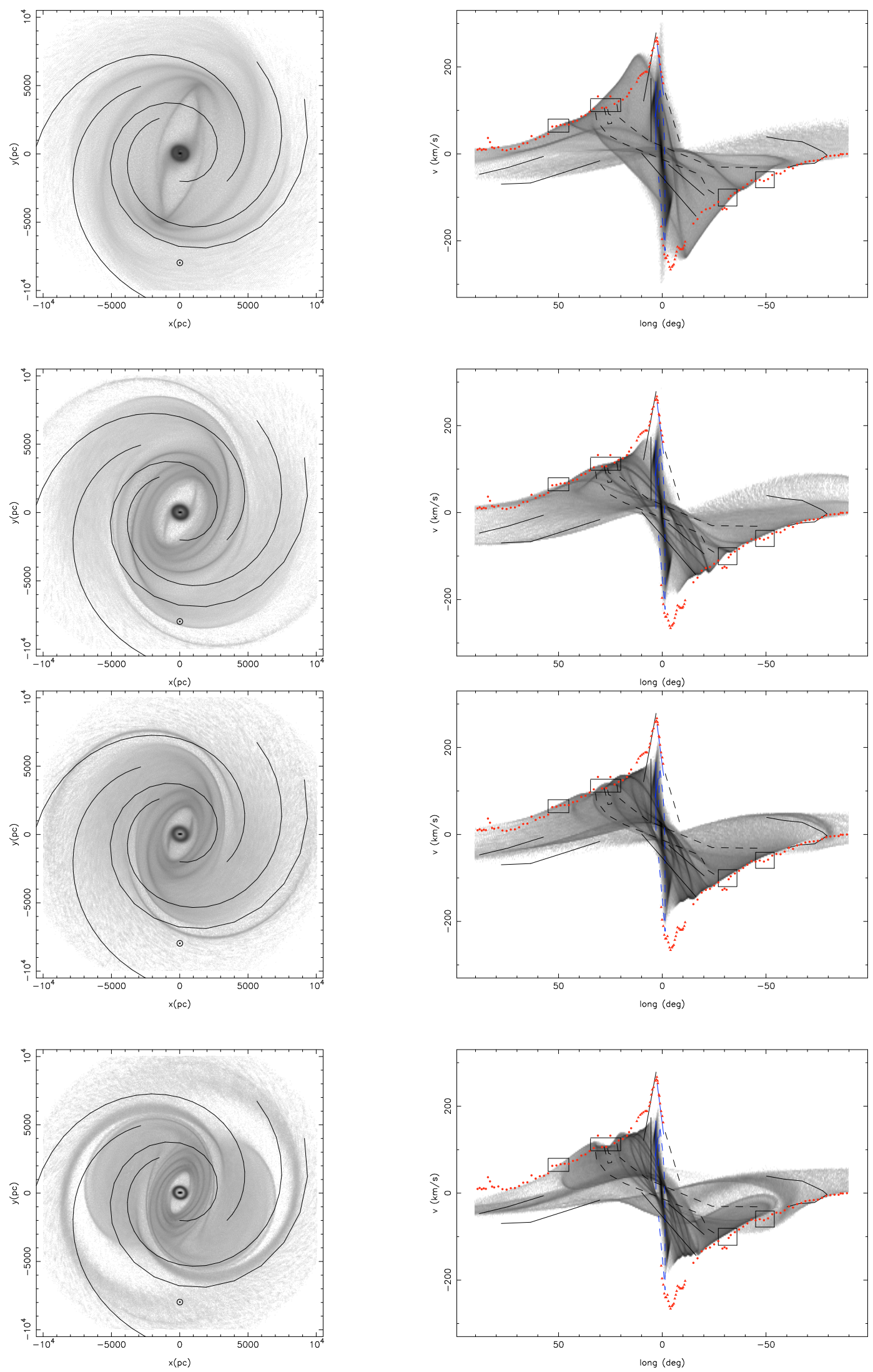

Fig. 8. Simulations for $\alpha^{B}=20^{\circ}$. The different rows represent the face-on view and the longitude velocity diagram for different pattern speeds of $20,30,40$ and $50 \mathrm{~km} \mathrm{~s}^{-1} \mathrm{kpc}^{-1}$ (from upper to lower rows). The solid lines represent the spiral arms as defined by Nakanishi \& Sofue (2006). The line Sun-GC first crosses the Sgr-Carina arm and the Scutum-Crux arm. In the other direction, the line of sight to the Galactic anticenter crosses the Perseus arm. The fourth arm is the Norma-Outer arm. 

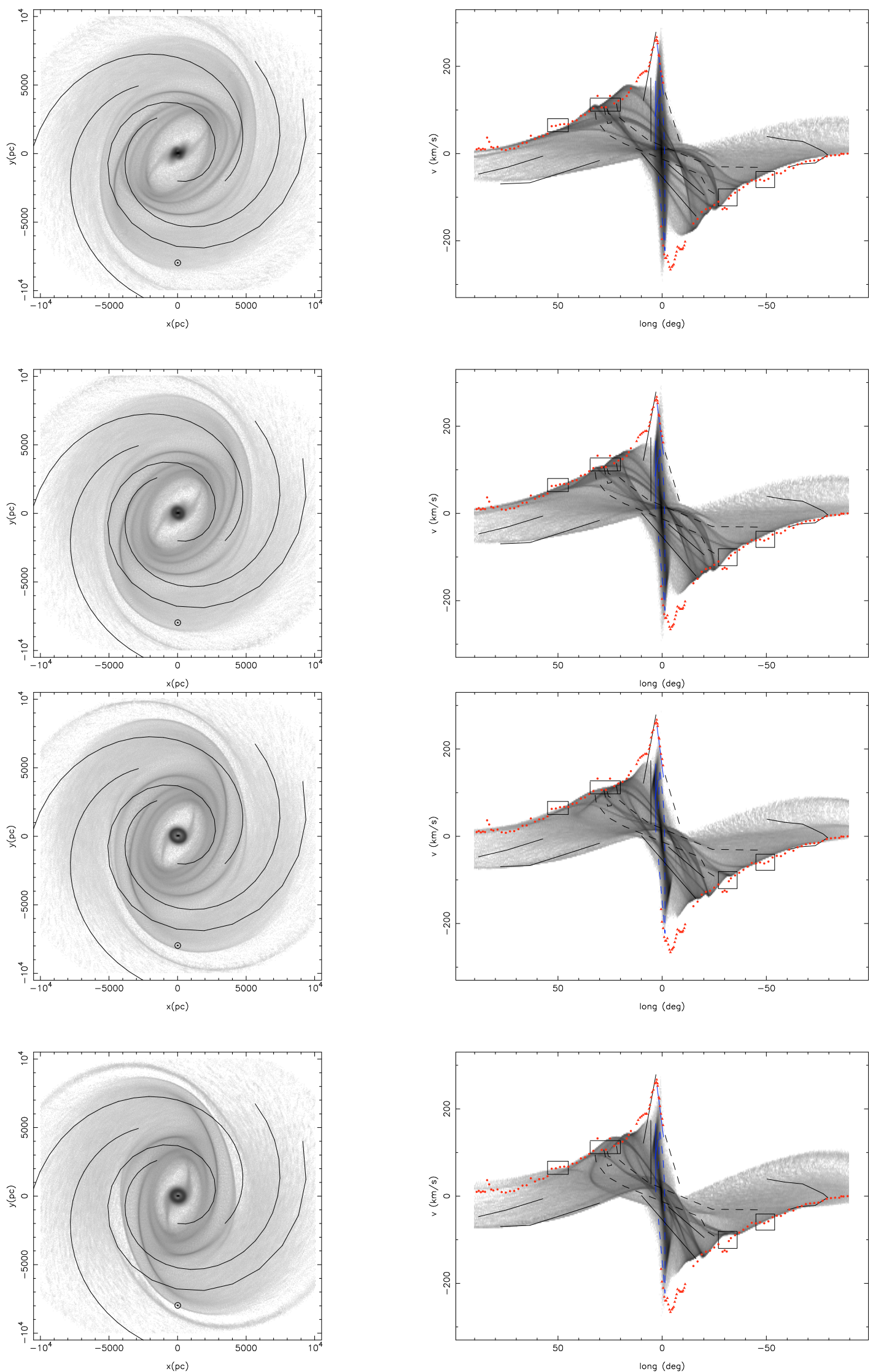

Fig. 9. Simulations for $\Omega_{\mathrm{p}}=30^{\circ}$. The different rows represent the face-on view and the longitude velocity diagram for different orientations of the bulge $\alpha^{B}$ of 45, 35, 20 and $10 \mathrm{deg}$ from the upper to the lower row. 
relatively slow bar models $\left(30-40 \mathrm{~km} \mathrm{~s}^{-1} \mathrm{kpc}^{-1}\right.$, Weiner \& Sellwood 1999; Fux 1999, this work) is comparable to several determinations of the spiral pattern speed that give values of 30-35 $\mathrm{km} \mathrm{s}^{-1} \mathrm{kpc}^{-1}$ (Mishurov \& Zenina 1999; Fernández et al. 2001; Lépine et al. 2001). On the other hand, Ibata \& Gilmore (1995) have measured a rotation speed of $25 \pm$ $4 \mathrm{~km} \mathrm{~s}^{-1} \mathrm{kpc}^{-1}$ for the bulge in the $0.7<R<3.5 \mathrm{kpc}$ region. Therefore, at present there are still many uncertainties regarding the speeds of the different patterns and it is not clear whether the spirals and the bar are decoupled or they turn at the same speeds. Simulations with an independent pattern speed for the spirals would be needed to study this question in detail but this is beyond the scope of the current paper, which is mainly devoted to the inner Galaxy.

\subsection{The GMR}

To obtain further insight into the structure and the dynamics of the Galaxy, we present below a more detailed analysis of the model with $\Omega_{\mathrm{p}}=30 \mathrm{~km} \mathrm{~s}^{-1} \mathrm{kpc}^{-1}$ and $\alpha^{B}=20^{\circ}$. We have selected different structures in the face-on view and we show the locus of the different structures in the $l v$-diagram (Fig. 10).

As already mentioned in Sect. 2.2, it is uncertain whether the GMR is an actual ring or it is composed by imbricated spiral arms. In our models the radius of the GMR (4-5 kpc) corresponds exactly with the position of the Ultra-Harmonic resonance for $\Omega \sim 30 \mathrm{~km} \mathrm{~s}^{-1} \mathrm{kpc}^{-1}$ (Fig. 7). However, we do not support the idea that the GMR is a resonant ring.

As shown in the upper panels of Fig. 10, in our models the GMR is composed of the inner parts of the spiral arms, which at those radii show almost circular motion. This is in agreement with the face-on views of the CO and HI data of Nakanishi \& Sofue (2006), who have proposed that the GMR is formed by the Scutum-Crux arm (near side of the GMR) and the inner part of the Sgr-Carina (far side of the GMR). Englmaier \& Gerhard (1999) discussed that if formed by imbricated arms, the GMR should be outside the corotation, assuming that there cannot exist significant circular orbits inside the corotation to produce a feature resembling a circular ring. However, this statement only holds for fast bars, for which all the region inside the corotation is dominated by the bar. In contrast, in our models with a relatively slow bar, the inner part of the spiral arms is inside the corotation radius and exhibits almost circular motion.

\subsection{The 3-kpc arm and its far side counterpart}

The lower panels of Fig. 10 show the locus of the lateral arms that surround the bar. The two dot-dashed lines in the $l v$-diagram are the fit to the 3-kpc arm and to its far-side counterpart as given by Dame \& Thaddeus (2008). As already mentioned, the locus of the lateral arms in the $l v$-diagram reproduce quite well the structure of the 3-kpc arm. This is in agreement with Fux (1999), who has already proposed that the 3-kpc arm could be a lateral arm. On the other hand, Fux (1999) proposed that the "135 $\mathrm{km} \mathrm{s}^{-1}$ arm" is the far side counterpart of the 3-kpc arm. However, our models predict a far side counterpart of the 3-kpc arm whose locus in the $l v$-diagram is almost symmetrical to the $3-\mathrm{kpc}$ arm. This is in perfect agreement with the recently found far 3-kpc arm (Dame \& Thaddeus 2008). The full implications of the new finding by Dame \& Thaddeus (2008) will be presented elsewhere.
The fact that the 3-kpc arms are lateral arms imply that they cannot be a channel to transport gas from the corotation to the CMZ as suggested by Habing et al. (2006), at least not directly.

\subsection{The off-axis arms or dustlanes}

In contrast to the $3-\mathrm{kpc}$ arms, the dustlanes do contribute to the transport of gas to the inner regions. In our models the locus of the dustlanes in the $l v$-diagram are vertical structures with velocities from $\sim 0 \mathrm{~km} \mathrm{~s}^{-1}$ to more than $100 \mathrm{~km} \mathrm{~s}^{-1}$. The maximum velocity increases as $l$ decreases. These vertical structures in the $l v$-diagram resemble the Clumps found by Bania (1977) and discussed recently by Liszt (2006, 2008). Nevertheless, our models do not reproduce the Galactic longitude of the observed Clumps $\left(5.5^{\circ}\right.$ and $\left.3.1^{\circ}\right)$. We confirm that Banias's Clumps are probably shocked gas in the dustlanes, which was the original interpretation of Stark \& Bania (1986) for the "Clump 2". The fact that the Clumps are composed of gas undergoing the dustlane shocks is also suggested by observational evidences, both kinematical (linewidths, velocity gradients, Stark \& Bania 1986; Liszt 2006) and chemical (Rodriguez-Fernandez et al. 2006). Taking into account the discrete appearance of Bania's clumps at two Galactic longitudes, it is likely that the dust lanes of the Milky Way are patchy, as is commonly observed in external galaxies.

Fux (1999) and Marshall et al. (2008) have proposed a slightly different interpretation. They propose that the Connecting Arm is the locus of the near side dustlane in the $l v$ diagram. However, the linewidths of the gas in the Connecting Arm do not show the velocity dispersion expected in the dustlane shocks (see for instance Reynaud \& Downes 1998). Indeed probably both Bania's clumps and the Connecting Arm are related to the dustlane. We reckon that Bania's Clumps are clouds that are undergoing the strong shocks expected in the dustlanes while the Connecting Arm is most likely the post-shocked and accelerated gas (see also the discussion in Liszt 2006). Indeed, in our simulations the maximum velocity of the gas in the dustlanes increases as $l$ decreases with the same steep of the Connecting Arm. Observationally, this interpretation is supported by the fact that the Clump at $l=5.5^{\circ}$ seems to be connected to to Connecting Arm at a negative latitude (see Bitran et al. 1997). The situation was less clear for "Clump 2" at $l=3.1^{\circ}$ since it is found at positive latitudes. However, this apparent problem has been solved with the recent discovery by Liszt (2008) of an emission feature with the same inclination in the $l v$-diagram as the Connecting Arm but at positive latitude. In addition it seems connected to Clump 2. In any case, the Clump 2 exhibits a rich shock chemistry as expected for gas in the dustlane shocks (Rodriguez-Fernandez et al. 2006)

\section{Simulation results and discussion: nuclear bar, $\mathrm{HI}$ ring and $\mathrm{CMZ}$}

Figure 11 shows the face-on view of the inner $2 \mathrm{kpc}$ of the modeled galaxies and the $l v$-diagrams for different orientations of the nuclear bar. All the other parameters are the same as those discussed in the previous section and the large scale face on views and $l v$-diagram s are the same, independent of the orientation of the nuclear bar.

The dustlanes end in a ring and inside this ring there is a small bar-like structure that is connected to the ring by two small spiral arms. The HI ring has an inner and outer radius of $300 \mathrm{pc}$ and $800 \mathrm{pc}$, respectively, in excellent agreement with the $\mathrm{HI}$ observations of Liszt \& Burton (1980) (see their Fig. 3). The ring 


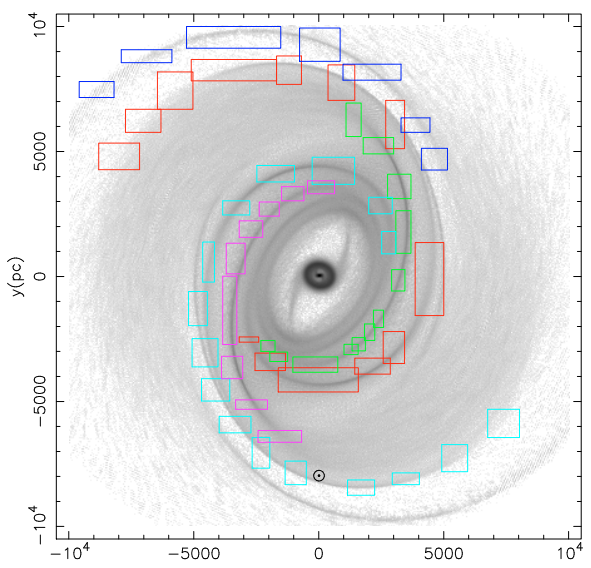

$x(p c)$

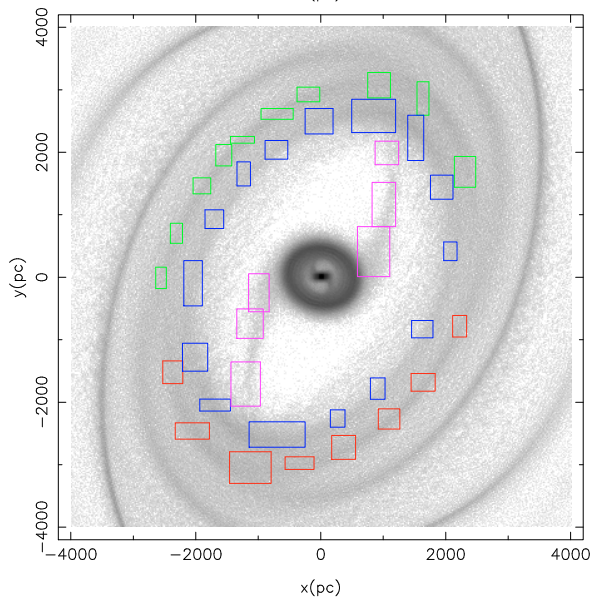

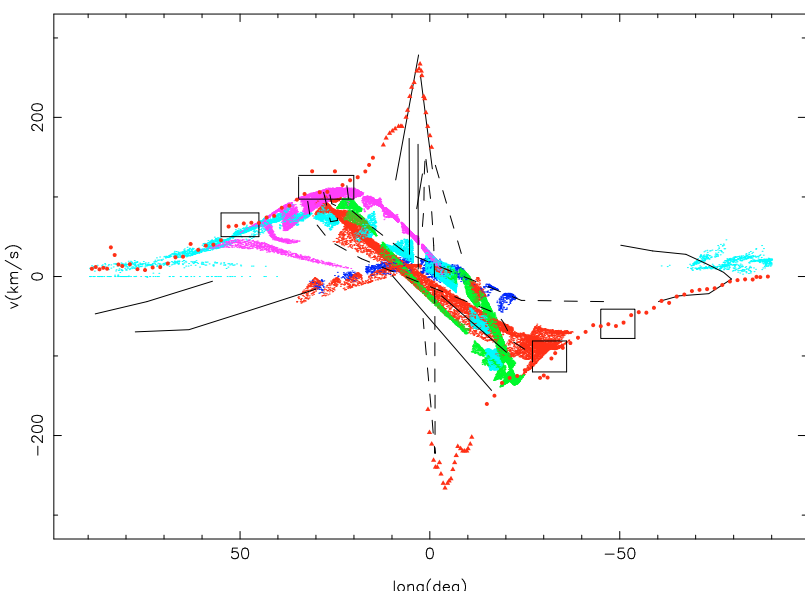

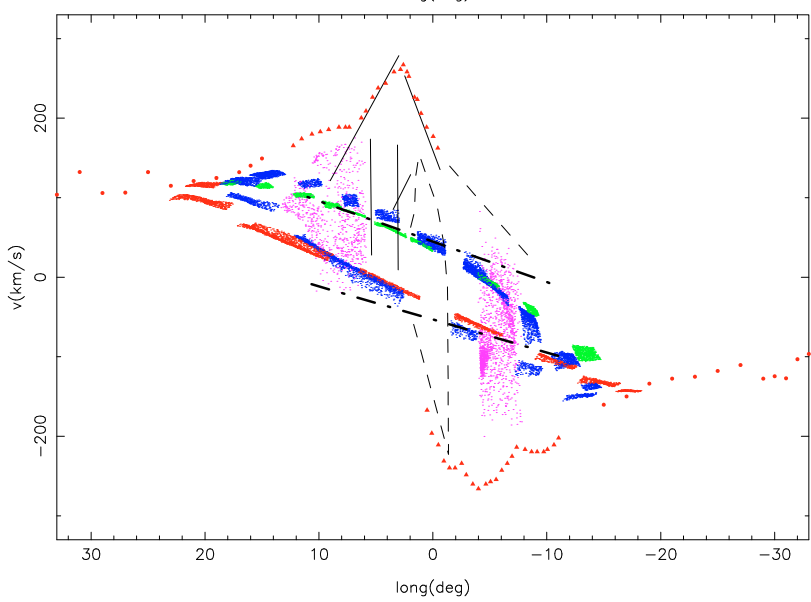

Fig. 10. Simulations for $\alpha^{B}=20^{\circ}$ and $\Omega_{\mathrm{p}}=30$. We have selected different regions in the face on views to identify the locus of the different structures in the $l v$-diagram (using the same color code in the face on view and the $l v$-diagram). Solid and dashed lines, circles and triangles are the same as in the previous figures. The dot-dashed lines in the lower $l v$-diagram are the fits to the near and far 3-kpc arms as given by Dame \& Thaddeus (2008).

is almost circular. In some simulations we have also found elliptical rings with the major axis approximately perpendicular to the large bar. Therefore, the ring is not supported by $X_{1}$ orbits elongated in the direction of the large bar (see a review of the different proposed models in Ferrière et al. 2007).

\subsection{The orientation of the nuclear bar}

In the central hundreds of parsecs there is an elongated structure that corresponds to the gas response to the nuclear bar and that resembles the observed $\mathrm{CMZ}$.

We have analyzed the shape of the $l v$-diagram as a function of the nuclear bar orientation. Figure 11 shows the $l v$-diagram obtained for nuclear bar orientations from $0^{\circ}$, to $15^{\circ}, 30^{\circ}$ and $45^{\circ}$. Our modeled $l v$-diagrams do not reproduce the components K and J of Rodriguez-Fernandez et al. (2006). In contrast, the $l v$-diagram of the $\mathrm{CMZ}$ is a parallelogram that resembles the observed one (Fig. 2). The inclination of the observed parallelogram is very well reproduced for an angle of $15^{\circ}$. After the Binney et al. (1991) paper, Jenkins \& Binney (1994) tried to reproduce the parallelogram discussed by Binney et al. (1991) using n-body simulations but with little success. To our knowledge, this is the first time that numerical simulations of the gas dynamics of the Milky Way naturally reproduce the parallelogram of the $l v$-diagram of the CMZ. This is probably due to the accurate potential that we have determined with the deep 2MASS data.
The orientation angle of the small bar inferred from our simulations is in excellent agreement with our models of the 2MASS star counts and with the face on view of the CMZ inferred from $\mathrm{CO}$ and OH data by Sawada et al. (2004). We conclude that the observed CMZ is most likely the gas response to the nuclear bar and that the orientation of the nuclear bar is $\alpha^{b} \sim 75^{\circ}$.

\subsection{The mass of the nuclear bar}

In the simulations discussed above, the velocity dispersion of the CMZ is well reproduced for a mass of the nuclear bar of $2.0 \times 10^{9} M_{\odot}$ (Sect. 5.1). However, the terminal velocities of the HI ring do not match the observed ones. For further insight into the nuclear bar mass we have increased its value in order to reproduce the terminal velocities of the HI ring. In these models, the shape of the different mass components is still given by the fits to the 2MASS star counts map (Tables 3-6). However, we have considered different scaling factors for the nuclear bar and for the bulge and disc. Thus, instead of Eq. (18), the total density is given by:

$\rho^{T}=f\left(\rho^{D}+\rho^{B}\right)+f^{b} \rho^{b}+\rho^{H}$

where $\rho^{H}$ is given by Eq. (19). Therefore, the only parameter that we change with respect to previous models is $f^{b}$. Figure 12 shows the rotation curve for $f^{b}=1.1 \times 10^{9}$, which gives a nuclear bar mass of $5.5 \times 10^{9} M_{\odot}$. All the other parameters 
N. J. Rodriguez-Fernandez and F. Combes: Gas flow models in the Milky Way embedded bars
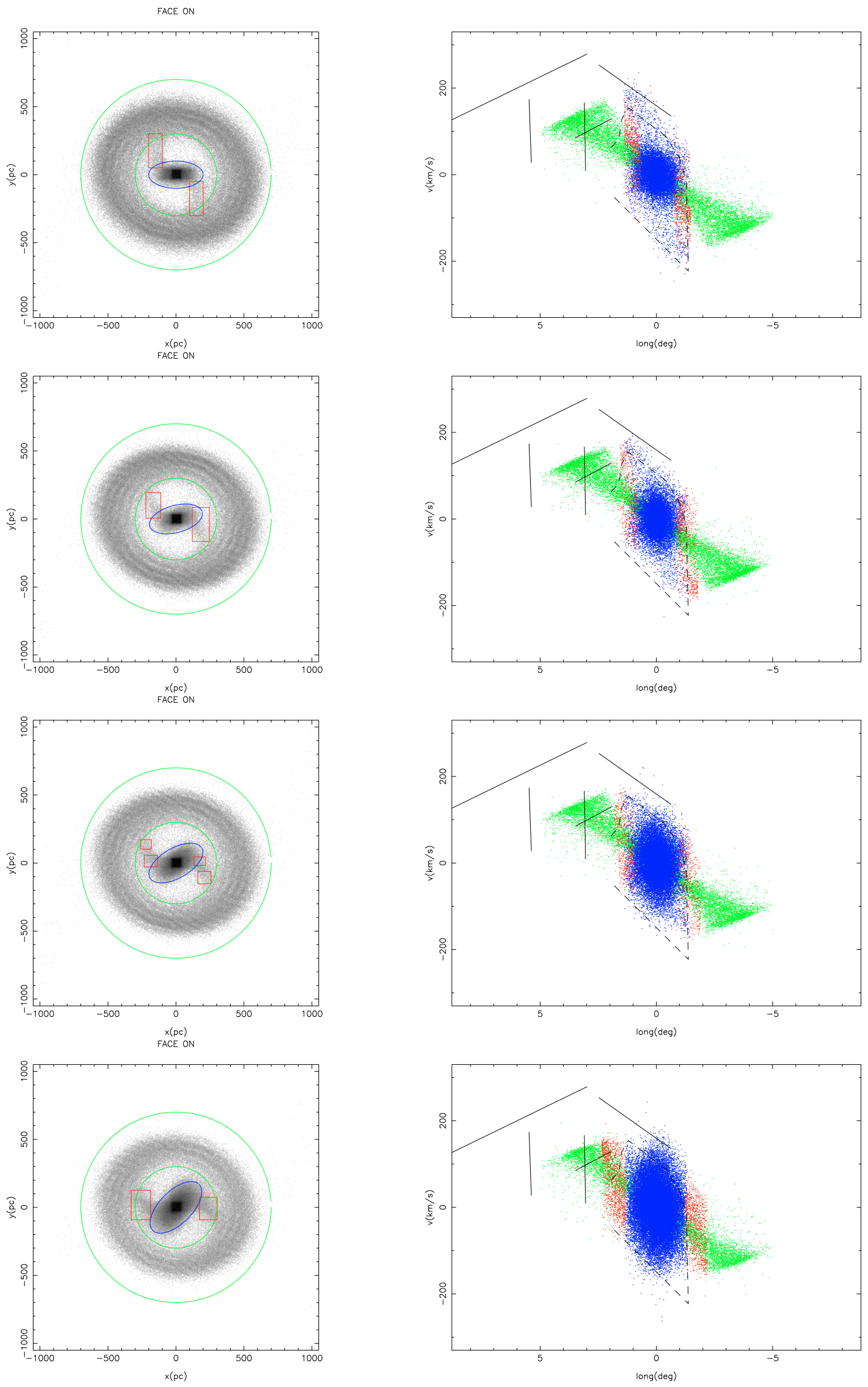

Fig. 11. Simulations for $\alpha^{B}=20^{\circ}, \Omega_{\mathrm{p}}=30$ and $\alpha^{b}=90^{\circ}, 75^{\circ}, 60^{\circ}$, and $45^{\circ}$ (from top to bottom). 


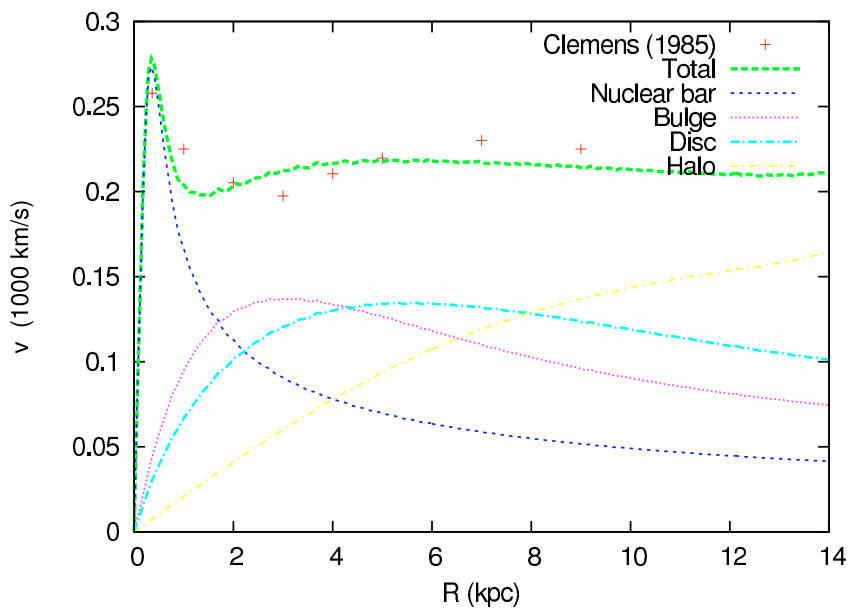

Fig. 12. Same as Fig. 6 but with a nuclear bar mass of $M^{b}=5.5 \times$ $10^{9} M_{\odot}$.

and masses are the same as those mentioned in Sect. 5.1. The simulation results for the inner $2 \mathrm{kpc}$ of the Galaxy are shown in Fig. 13. The face on view is characterized by a large ring with an inner and outer radius of 200 and $1200 \mathrm{pc}$, respectively. The $l v$-diagram of this ring reproduces the terminal velocity of the HI observations, which at positive longitude coincides with the Connecting Arm. Indeed, Liszt \& Burton (1980) proposed that the Connecting Arm could be the edge of the HI ring.

It is difficult to reproduce both the terminal velocities of the $\mathrm{CMZ}$ and $\mathrm{HI}$ ring, however from the previous considerations we conclude that the mass of the nuclear bar should be in the range $(2-5.5) \times 10^{9} M_{\odot}$.

\subsection{Is the nuclear bar lopsided?}

The nuclear bar in the star count map of Alard (2001) seems to be shifted towards negative longitudes (in contrast to the gas distribution that seems to be shifted towards positive longitudes). Our star count model shows that fits with a lopsided nuclear bar have a $\chi^{2}$ that is $9 \%$ lower than the $\chi^{2}$ for models that do not allow a lopsided bar. The symmetry center of the bar could be shifted by 22-25 pc with respect to the dynamical center of the Galaxy.

We have studied the gas response to a lopsided nuclear bar in order to investigate whether the observed asymmetry of the CMZ could be explained by a lopsided nuclear bar as inferred from the 2MASS data. As discussed by Morris \& Serabyn (1996) the signature of a $m=1$ mode in the Galactic center would be a shift of the gas distribution in $l$ but also a shift of the velocity centroid of the $l v$-diagram.

Figure 14 shows a simulation of the gas flow in the potential computed from the fit to the 2MASS star counts map with $\alpha^{b}=75^{\circ}$ (for which $x_{1}=0.023 \mathrm{kpc}$ ). The $l v$-diagram of the gas shows a parallelogram whose sides show the good inclination. The simulated parallelogram is only slightly asymmetric in $l$ and $v$. To better understand the effect of a lopsided nuclear bar, we have also computed a simulation with the same nuclear bar but artificially shifted by $90 \mathrm{pc}$ along its major axis towards the third Galactic quadrant $\left(x_{1}=0.090 \mathrm{kpc}\right)$. The results are also shown in Fig. 14. The $l v$-diagram is still only slightly asymmetric in $v$ but now it is clearly asymmetric in $l$. The gas response to the lopsided stellar bar follows the star distribution and it is lopsided towards negative longitudes as well. To obtain an $l v$-diagram with a parallelogram similar to that of the CO data shown in Fig. 2, the nuclear bar should be lopsided towards the first quadrant. This is shown in Fig. 14, where $x_{1}=-0.090 \mathrm{kpc}$. However, such a bar is not compatible with the 2MASS star counts.

We conclude that, both from the fits to the 2MASS data and from the numerical simulations of the gas dynamics, there is no clear evidence of an intrinsic lopsidedness in the stellar potential. The observed asymmetry of the gas distribution in the CMZ cannot be explained as the gas response to a lopsided nuclear bar in the way suggested by Alard's map.

\section{Discussion}

\subsection{The origin of the asymmetry of the $C M Z$}

Although the origin of the asymmetry of the CMZ is a longstanding problem, it has not been discussed much in the literature. Two exceptions are the papers by Combes (1996) and Morris \& Serabyn (1996). The first paper proposed that the observed asymmetry could be due to a $m=1$ mode of the potential since lopsidedness is commonly observed in the central regions of external galaxies. The second paper discusses how the effect of the presence of a $m=1$ mode in the Galactic center would be an asymmetry of the CMZ both in longitude and in velocity. However, we have shown in the previous section that the possible lopsidedness of the stellar distribution cannot explain the observed asymmetry of the CMZ. Furthermore, there is no clear evidence of an intrinsic asymmetry of the stellar potential. Regarding the gas, the distribution is clearly asymmetric in $l$ but the parallelogram of Fig. 2 is rather symmetric in $v$. In addition, the structures found by Sofue (1995) that seem to be the signature of a quasi-circular ring inside the $\mathrm{CMZ}$ and that could be the nuclear bar equivalent to the HI ring, do not show an asymmetry in $v$.

Indeed our simulations propose an alternative explanation for the asymmetric distribution of the CMZ in which the stellar potential in the GC is strictly symmetric. The simulations show that the HI ring can be connected to the CMZ by a pair of small spiral arms. For instance, the simulation with $\alpha^{b}=75$ (second row of Fig. 11) shows that the clouds in the arm seen at negative longitudes will be seen in the $l v$-diagram outside of the observed parallelogram while the clouds in the arm seen at positive longitudes "fill" the observed parallelogram at the position of the $l=1.3^{\circ}$-complex. Observations of the molecular gas in external galaxies show that, in contrast to most numerical simulations, the gas distribution in real galaxies is rarely symmetric. This could also be the case in the Milky Way. The apparent asymmetry of the $\mathrm{CMZ}$ can be due to material falling into the CMZ from the $\mathrm{HI}$ ring through only one of the inner spiral arms. In this context, strong shocks are expected in the interaction region: the $l=1.3^{\circ}$-complex .

Observationally, the $l=1.3^{\circ}$-complex looks very different to the Sgr's cloud complexes. For instance, in the $l b$ map of Fig. 2, it is clear that the gas distribution from $\mathrm{Sgr} \mathrm{C}$ to $\mathrm{Sgr} \mathrm{B}$ is rather symmetric and thin. The asymmetry in $l$ is indeed exclusively due to the $l=1.3^{\circ}$-complex. Furthermore, the $l=1.3^{\circ}$-complex shows an unusual latitude extension, much higher that the rest of the CMZ. The velocity structure of this cloud complex is also unusual, showing a wide velocity dispersion (the $l=1.3^{\circ}$-complex is indeed the most prominent feature in the $l v$-diagram). The chemistry also gives us interesting hints as to the nature of this cloud complex. On the one hand, Hüttemeister et al. (1998) have found that the highest abundances of the shock tracer molecule $\mathrm{SiO}$ are found at one of the extremities of the CMZ: in the $l=1.3^{\circ}$-complex. They have already proposed that this cloud 

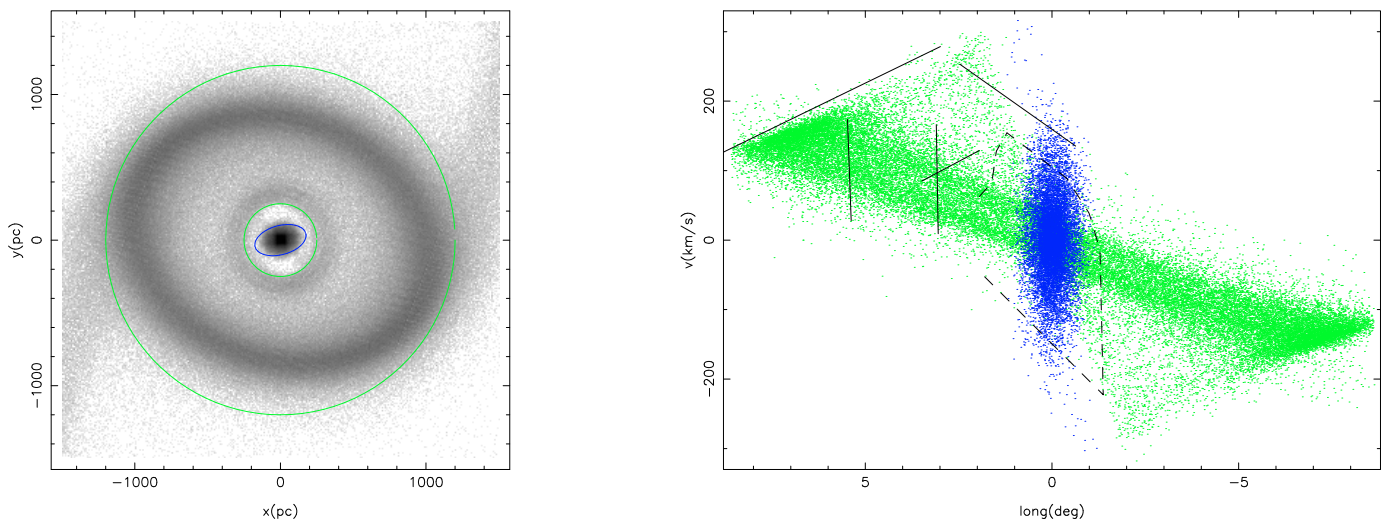

Fig. 13. Simulations results for $\alpha^{B}=20^{\circ}, \Omega_{\mathrm{p}}=30$ and $\alpha^{b}=75^{\circ}$ (with nuclear bar mass of $25 \%$ of bulge mass).
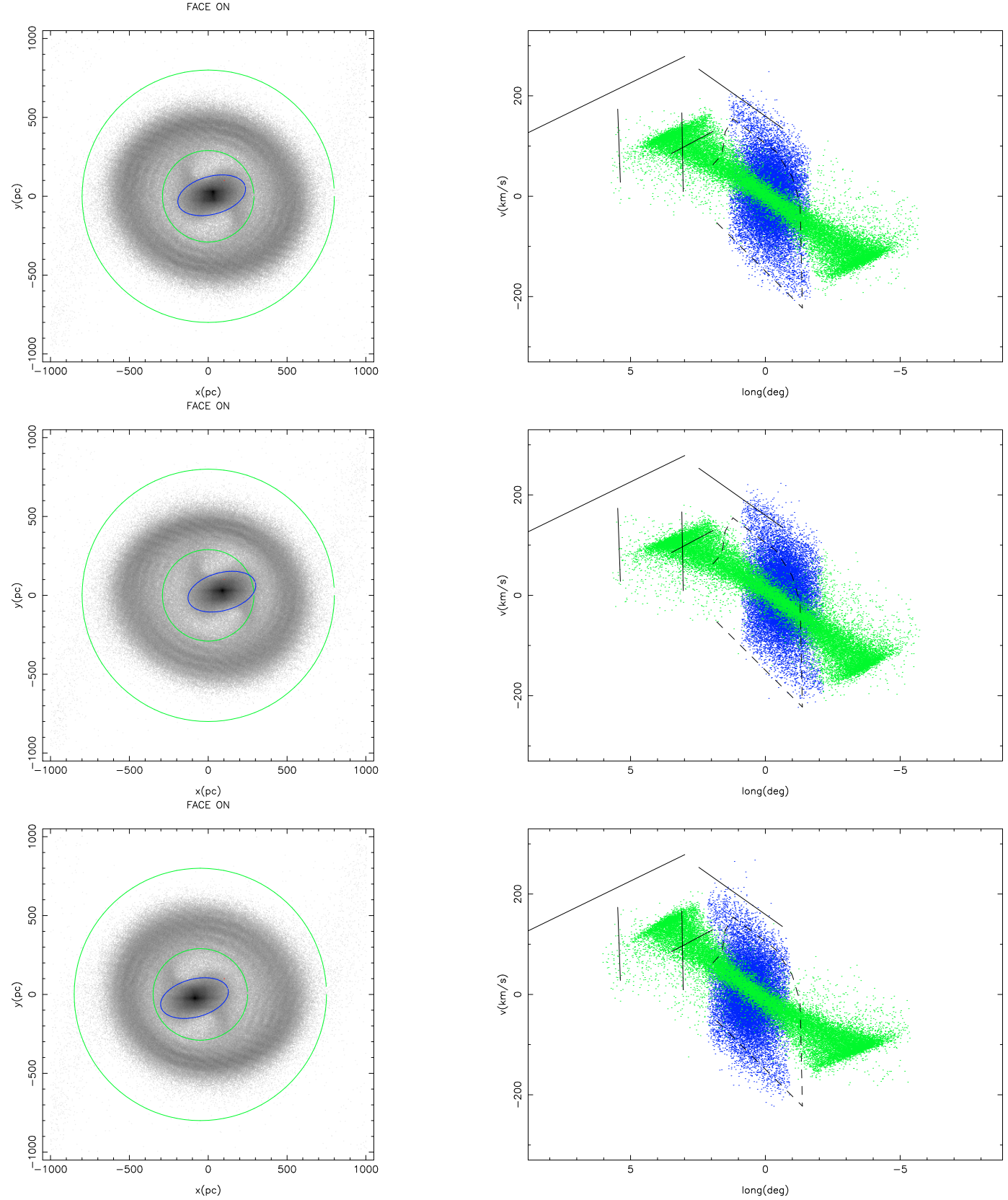

Fig. 14. Simulations for $\alpha^{B}=20^{\circ}, \Omega_{\mathrm{p}}=30$ and $\alpha^{b}=75^{\circ}$. The upper panel corresponds to the best fit to the 2MASS data with a lopsided bar $\left(x_{1}=23 \mathrm{pc}\right)$. The middle panel is a simulation with the same nuclear bar but $x_{1}=90 \mathrm{pc}$ and the lower panel is a simulation with the same nuclear bar but $x_{1}=-9$. 
complex represents the scenario of strong shocks due to gas falling into the CMZ. On the other hand, Rodriguez-Fernandez et al. (2006) have presented a more complete study of the $\mathrm{SiO}$ emission in all the kinematical structures of the GC. They have detected $\mathrm{SiO}$ in at least one cloud of each kinematical structure. However, the only feature where they detected high abundances of $\mathrm{SiO}$ in every observed cloud is the $l=1.3^{\circ}$-complex. In agreement with Hüttemeister et al. (1998); Rodriguez-Fernandez et al. (2006) have measured the highest $\mathrm{SiO}$ abundances in this region. Therefore, the $l b$ distribution, the velocity structure, and the chemistry imply that the cloud complex giving rise to the observed asymmetry of the CMZ (the $l=1.3^{\circ}$-complex) shows significant differences to other CMZ complexes. In particular, it shows the signature of strong shocks. Therefore, we suggest that the observed asymmetry of the CMZ can be the result of gas falling into the CMZ and not due to an intrinsic asymmetry of the stellar potential. This explanation is also supported by our detailed simulations of the stellar structure and the gas dynamics.

\subsection{The nature of the nuclear bar}

Double bars are commonly observed in external galaxies (Shaw et al. 1993; Wozniak et al. 1995; Elmegreen et al. 1996). Around $28 \%$ of barred galaxies have a secondary bar (Erwin \& Sparke 2002; Laine et al. 2002). Nuclear or secondary bars are objects of prime interest to explain the gas inflow towards the center of a galaxy and the fueling of Active Galactic Nuclei (Shlosman et al. 1989).

Several works have studied the stellar orbits that support nested bars (Maciejewski \& Sparke 1997, 2000) and their stability (El-Zant \& Shlosman 2003). In addition, double barred galaxies have been the subject of a number of numerical simulations of star and gas dynamics that have shown a plethora of phenomena, such as: i) secondary bars forming in purely stellar discs (Rautiainen et al. 2002) or only in the presence of gas (Combes 1994); ii) secondary bars forming before (Rautiainen \& Salo 1999) or after the primary bars (Combes 1994; Englmaier \& Shlosman 2004; Heller et al. 2007); iii) decoupled secondary bars rotating slower (Heller et al. 2001) or faster (Friedli \& Martinet 1993; Combes 1994; Rautiainen et al. 2002; Englmaier \& Shlosman 2004) than the primary bar; iv) two misaligned bars rotating at the same speed (Shaw et al. 1993; Combes 1994).

The secondary bars rotating at the same speed as the primary bars have been explained by Shaw et al. (1993) and Combes (1994) as follows: the gas clouds tend to shift from the closed $X_{1}$ orbits to the perpendicular $X_{2}$ orbits of the primary bar. However, dissipative collisions between the gas clouds reduce the orthogonality of this phase shift and the gas settles in a leading phaseshifted bar. If the gas fraction is high, its gravitational influence is then sufficient to modify the stellar component and to form a secondary bar that is still coupled to the primary bar. If the secondary and primary bars remain coupled and they continue rotating with the same speed, the bars will align or form an angle of $90^{\circ}$ since the primary bar exerts a gravitational torque on the secondary bar and vice-versa (Friedli \& Martinet 1993). Alternatively, the secondary bar may increase its rotation speed. Thus, the coupled phase can be a precursor of a future decoupled phase. The dynamical process from coupled to decoupled gaseous nuclear bars has been studied by Heller et al. (2001); Englmaier \& Shlosman (2004) and, more recently, by Heller et al. (2007), who have carried out the first simulations of the formation of nested bars from cosmological initial conditions. In these simulations a secondary bar forms in response to the gas inflow along the stellar primary bar. This gaseous bar is initially corotating with the primary bar. Subsequent mass inflow strengthens the bar giving rise to even more rapid gas inflow. During this process the secondary bar shrinks in size and the pattern speed increases at a rate inversely proportional to the bar size. The pattern speeds of the two bars are such that the secondary bar corotation coincides with the primary bar inner Lindblad resonance, suggesting that non-linear interactions between the two bars are at play (Tagger et al. 1987).

Our simulations are the very first attempt to model the Milky Way with two nested bars and have been performed assuming a common pattern speed for both bars. Therefore, we cannot study in detail the coupling and the evolution of the nuclear bar. Nevertheless, these simulations explain many characteristics of the Galaxy at scales from the disk to the nuclear region, some for the first time, such as the parallelogram of the CMZ. Therefore, the observational data are compatible with a scenario of coupled bars rotating with the same speed. Our results imply that the nuclear bar is leading the large primary bar by $\sim 55^{\circ}$, as expected in this context (Combes 1994). Thus, the decoupling of the nuclear bar can still not be effective in the Milky Way. The dynamical decoupling of the two bars will be the subject of a forthcoming paper.

\section{Conclusions}

We have presented gas flow models in the mass distribution derived from 2MASS star counts using a model with three components (disk, bulge and nuclear bar). Our dynamical models are the first ones that include a central mass cusp (the nuclear bar) constrained by observations. For the first time, we have obtained good models of the Milky Way from the scales of the spiral arms to the Central Molecular Zone (CMZ) in the Galactic center (GC). The best models are found for a bulge orientation of $20-35^{\circ}$ with respect to the Sun-GC line and a pattern speed of $30-40 \mathrm{~km} \mathrm{~s}^{-1} \mathrm{kpc}^{-1}$. This places the corotation radius at 5-7 kpc. The simulations reproduce:

- The spiral arms, giving in particular a good tangent point for the Carina arm.

- The Galactic Molecular Ring (GMR), which is not an actual ring but the inner parts of the spiral arms and it is found approximately at the position of the ultra-harmonic resonance.

- The 3-kpc arm and its far-side counterpart, which are the lateral arms that surround the bar.

- The HI ring, with a very similar size to that derived from the observations.

- The Central Molecular Zone (CMZ).

In addition, we postulate the existence of small spiral arms arising from the extremities of the $\mathrm{CMZ}$ and linking the $\mathrm{HI}$ ring.

In our simulations, the $\mathrm{CMZ}$ is the gas response to the nuclear bar. These simulations reproduce, for the first time, the parallelogram shape of the $l v$-diagram of the CMZ. Using this shape we have been able to constrain the nuclear bar orientation, which is of $\sim 60^{\circ}-75^{\circ}$ with respect to the Sun-GC line, in excellent agreement with the 2MASS fits and the results of Sawada et al. (2004). We have also studied the observed asymmetry of the $\mathrm{CMZ}$ and conclude that it cannot be due to the lopsidedness of the nuclear bar as suggested by the 2MASS maps. Gas dynamical simulations in a lopsided potential cannot reproduce the observations, and we do not see strong direct evidence in the 2MASS data of an intrinsic lopsidedness of the stellar potential.

We propose an alternative scenario to explain the observed asymmetry of the CMZ. The asymmetry can be due to gas 
falling into the CMZ through the inner spiral arm in the $l=1.3^{\circ}$-complex region. We have also discussed that the $l=$ $1.3^{\circ}$-complex shows significant differences with the, otherwise symmetric, $\mathrm{CMZ}$ that extends from Sgr C to Sgr B. In particular, the $l=1.3^{\circ}$-complex shows all the signatures of the shocks expected if it is an interaction region where new material is falling into the CMZ.

In our models both bars rotate with the same pattern speed. The success of these models in explaining many characteristics of the Milky Way, such as the Central Molecular Zone (CMZ) imply that the observations are compatible with that assumption. Furthermore, in our models the nuclear bar leads the large bar by $\sim 55^{\circ}$ as expected for coupled nested bars (Combes 1994). Therefore, in spite of the high mass of the nuclear bar (10-25\% of the mass of the bulge) the dynamical decoupling my still not be effective. However, this question requires further studies and gas flow models with two pattern speeds or self-consistent gas and star dynamics simulations.

Acknowledgements. We thank Katia Ferrière for a critical reading of the manuscript and Tom Dame for sharing with us his finding of the far-side counterpart of the 3-kpc arm before publication. We also thank the anonymous referee for useful comments that have improved the paper, in particular regarding the phenomena of several pattern speeds and nested bars in other galaxies. The early stages of this work have benefited from support by a Marie Curie Fellowship of the European Community program "Improving Human Research Potential and the Socio-economic Knowledge base" under contract number HPMF-CT-200201677.

\section{References}

Alard, C. 2001, A\&A, 379, L44

Alvarez, H., May, J., \& Bronfman, L. 1990, ApJ, 348, 495

Amaral, L. H., \& Lepine, J. R. D. 1997, MNRAS, 286, 885

Babusiaux, C., \& Gilmore, G. 2005, MNRAS, 358, 1309

Bally, J., Stark, A. A., Wilson, R. W., \& Henkel, C. 1987, ApJS, 65, 13

Bania, T. M. 1977, ApJ, 216, 381

Bash, F. N. 1981, ApJ, 250, 551

Benjamin, R. A. 2008, A\&AS, 212, 86.01

Benjamin, R. A., Churchwell, E., Babler, B. L., et al. 2005, ApJ, 630, L149

Binney, J., \& Merrifield, M. 1998, Galactic astronomy (Princeton University Press)

Binney, J., Gerhard, O. E., Stark, A. A., Bally, J., \& Uchida, K. I. 1991, MNRAS, 252,210

Binney, J., Gerhard, O., \& Spergel, D. 1997, MNRAS, 288, 365

Bissantz, N., \& Gerhard, O. 2002, MNRAS, 330, 591

Bissantz, N., Englmaier, P., \& Gerhard, O. 2003, MNRAS, 340, 949

Bitran, M., Alvarez, H., Bronfman, L., May, J., \& Thaddeus, P. 1997, A\&AS, 125,99

Blitz, L., \& Spergel, D. N. 1991, ApJ, 379, 631

Burke, B. F., \& Tuve, M. A. 1964, 20, Australian Academy of Science, Canberra, 183

Burton, W. B., \& Liszt, H. S. 1978, ApJ, 225, 815

Burton, W. B., \& Liszt, H. S. 1993, A\&A, 274, 765

Casoli, F., \& Combes, F. 1982, A\&A, 110, 287

Clemens, D. P. 1985, ApJ, 295, 422

Combes, F. 1994, in Proc. Mass-Transfer Induced Activity in galaxies, ed. I. Shlosman (Cambridge: Cambridge University Press), 170

Combes, F. 1996, Proc. 169th Symp. Inter. Astron. Union, held in The Hague, 169,133

Combes, F., \& Gerin, M. 1985, A\&A, 150, 327

Combes, F., \& Sanders, R. H. 1981, A\&A, 96, 164

Combes, F., Debbasch, F., Friedli, D., \& Pfenniger, D. 1990, A\&A, 233, 82

Dame, T., \& Thaddeus, P. 2008, ApJ, submitted

Dame, T. M., Hartmann, D., \& Thaddeus, P. 2001, ApJ, 547, 792

Debattista, V. P., Gerhard, O., \& Sevenster, M. N. 2002, MNRAS, 334, 355

Drimmel, R. 2000, A\&A, 358, L13

Drimmel, R., \& Spergel, D. N. 2001, ApJ, 556, 181

Dwek, E., Arendt, R. G., Hauser, M. G., et al. 1995, ApJ, 445, 716

Eaton, N., Adams, D. J., \& Giles, A. B. 1984, MNRAS, 208, 241

El-Zant, A. A., \& Shlosman, I. 2003, ApJ, 595, L41

Elmegreen, D. M., Elmegreen, B. G., Chromey, F. R., Hasselbacher, D. A., \& Bissell, B. A. 1996, AJ, 111, 1880
Englmaier, P., \& Gerhard, O. 1999, MNRAS, 304, 512

Englmaier, P., \& Shlosman, I. 2004, ApJ, 617, L115

Erwin, P., \& Sparke, L. S. 2002, AJ, 124, 65

Fernández, D., Figueras, F., \& Torra, J. 2001, A\&A, 372, 833

Ferrière, K., Gillard, W., \& Jean, P. 2007, A\&A, 467, 611

Fich, M., Blitz, L., \& Stark, A. A. 1989, ApJ, 342, 272

Freudenreich, H. T. 1998, ApJ, 492, 495

Friedli, D., \& Martinet, L. 1993, A\&A, 277, 27

Fux, R. 1999, A\&A, 345, 787

Garcia-Burillo, S., Sempere, M. J., Combes, F., \& Neri, R. 1998, A\&A, 333, 864 Georgelin, Y. M., \& Georgelin, Y. P. 1976, A\&A, 49, 57

Gerin, M., Casoli, F., \& Combes, F. 1991, A\&A, 251, 32

Habing, H. J., Sevenster, M. N., Messineo, M., van de Ven, G., \& Kuijken, K. 2006, A\&A, 458, 151

Heller, C., Shlosman, I., \& Englmaier, P. 2001, ApJ, 553, 661

Heller, C. H., Shlosman, I., \& Athanassoula, E. 2007, ApJ, 657, L65

Hohl, F., \& Hockney, R. W. 1969, J. Computat. Phys., 4, 306

Hüttemeister, S., Dahmen, G., Mauersberger, R., et al. 1998, A\&A, 334, 646

Ibata, R. A., \& Gilmore, G. F. 1995, MNRAS, 275, 605

Jenkins, A., \& Binney, J. 1994, MNRAS, 270, 703

Kerr, F. J. 1967, Radio Astronomy and the Galactic System, 31, 239

Kulkarni, S. R., Heiles, C., \& Blitz, L. 1982, ApJ, 259, L63

Laine, S., Shlosman, I., Knapen, J. H., \& Peletier, R. F. 2002, ApJ, 567, 97

Lee, C. W., Lee, H. M., Ann, H. B., \& Kwon, K. H. 1999, ApJ, 513, 242

Lépine, J. R. D., Mishurov, Y. N., \& Dedikov, S. Y. 2001, ApJ, 546, 234

Lin, C. C., Yuan, C., \& Shu, F. H. 1969, ApJ, 155, 721

Liszt, H. S. 2006, A\&A, 447, 533

Liszt, H. S. 2008, A\&A, submitted

Liszt, H. S., \& Burton, W. B. 1978, ApJ, 226, 790

Liszt, H. S., \& Burton, W. B. 1980, ApJ, 236, 779

Lopez-Corredoira, M., Garzon, F., Hammersley, P., Mahoney, T., \& Calbet, X. 1997, MNRAS, 292, L15

López-Corredoira, M., Hammersley, P. L., Garzón, F., Simonneau, E., \& Mahoney, T. J. 2000, MNRAS, 313, 392

López-Corredoira, M., Hammersley, P. L., Garzón, F., et al. 2001, A\&A, 373, 139

López-Corredoira, M., Cabrera-Lavers, A., \& Gerhard, O. E. 2005, A\&A, 439, 107

López-Corredoira, M., Cabrera-Lavers, A., Mahoney, T. J., et al. 2007, AJ, 133, 154

Maciejewski, W., \& Sparke, L. S. 1997, ApJ, 484, L117

Maciejewski, W., \& Sparke, L. S. 2000, MNRAS, 313, 745

Marshall, D. J., Fux, R., Robin, A. C., \& Reylé, C. 2008, A\&A, 477, L21

Martos, M., Hernandez, X., Yáñez, M., Moreno, E., \& Pichardo, B. 2004, MNRAS, 350, L47

Mishurov, Y. N., \& Zenina, I. A. 1999, A\&A, 341, 81

Morgan, S. 1990, PASP, 102, 102

Morris, M., \& Serabyn, E. 1996, ARA\&A, 34, 645

Mulder, P. S., \& Combes, F. 1996, A\&A, 313, 723

Mulder, W. A., \& Liem, B. T. 1986, A\&A, 157, 148

Nakanishi, H., \& Sofue, Y. 2003, PASJ, 55, 191

Nakanishi, H., \& Sofue, Y. 2006, PASJ, 58, 847

Nishiyama, S., Nagata, T., Baba, D., et al. 2005, ApJ, 621, L105

Picaud, S., Cabrera-Lavers, A., \& Garzón, F. 2003, A\&A, 408, 141

Press, W. H., Teukolsky, S. A., Vetterling, W. T., \& Flannery, B. P. 1992

Rautiainen, P., \& Salo, H. 1999, A\&A, 348, 737

Rautiainen, P., Salo, H., \& Laurikainen, E. 2002, MNRAS, 337, 1233

Reynaud, D., \& Downes, D. 1998, A\&A, 337, 671

Rieke, G. H., \& Lebofsky, M. J. 1985, ApJ, 288, 618

Rodriguez-Fernandez, N. J., Combes, F., Martin-Pintado, J., Wilson, T. L., \& Apponi, A. 2006, A\&A, 455, 963

Rougoor, G. W., \& Oort, J. H. 1960, Proceedings of the National Academy of Science, 46,1

Sawada, T., Hasegawa, T., Handa, T., \& Cohen, R. J. 2004, MNRAS, 349, 1167 Scoville, N. Z. 1972, ApJ, 175, L127

Sellwood, J. A., \& Sparke, L. S. 1988, MNRAS, 231, 25P

Sevenster, M. N. 1999, MNRAS, 310, 629

Shaw, M. A., Combes, F., Axon, D. J., \& Wright, G. S. 1993, A\&A, 273, 31

Shlosman, I., Frank, J., \& Begelman, M. C. 1989, Nature, 338, 45

Sofue, Y. 1995, PASJ, 47, 551

Stark, A. A., \& Bania, T. M. 1986, ApJ, 306, L17

Tagger, M., Sygnet, J. F., Athanassoula, E., \& Pellat, R. 1987, ApJ, 318, L43

Wainscoat, R. J., Cohen, M., Volk, K., Walker, H. J., \& Schwartz, D. E. 1992, ApJS, 83, 111

Weiner, B. J., \& Sellwood, J. A. 1999, ApJ, 524, 112

Wozniak, H., Friedli, D., Martinet, L., Martin, P., \& Bratschi, P. 1995, A\&AS, 111,115 
N. J. Rodriguez-Fernandez and F. Combes: Gas flow models in the Milky Way embedded bars, Online Material p 1

Table 3. Results of the fits to the star count data of Fig. 5 using a disc, bulge and nuclear bar. The following parameters have been taken from Table 1 and kept fixed: $\alpha^{B}=10, x_{0}^{B}=1.117, y_{0}^{B}=0.513, z_{0}^{B}=0.362 h_{\mathrm{r}}=2.5, h_{z}=0.153$. The bar angle $\alpha^{b}$ is also fixed, the other parameters are free and used to obtain the best fit to the data. The first set of results have been obtained with $x_{1}$ fixed to 0 . In the second set of results $x_{1}$ is a free parameter.

\begin{tabular}{lllllllll}
\hline \hline Bulge & Disk & \multicolumn{7}{c}{ Bar } \\
$\rho_{0}^{B}$ & $\rho_{0}^{D}$ & $x_{0}^{b}$ & $y_{0}^{b}$ & $z_{0}^{b}$ & $\rho_{0}^{b}$ & $\alpha^{b}$ & $x_{1}$ & $\chi^{2}$ \\
& & $\mathrm{kpc}$ & $\mathrm{kpc}$ & $\mathrm{kpc}$ & & $\mathrm{deg}$ & $\mathrm{kpc}$ & \\
\hline $0.379 \mathrm{E}-02$ & $0.257 \mathrm{E}-03$ & 0.195 & 0.146 & 0.103 & 0.103 & 165.0 & 0.0 & 0.374 \\
$0.380 \mathrm{E}-02$ & $0.256 \mathrm{E}-03$ & 0.189 & 0.144 & 0.102 & 0.113 & 150.0 & 0.0 & 0.367 \\
$0.381 \mathrm{E}-02$ & $0.256 \mathrm{E}-03$ & 0.175 & 0.138 & 0.100 & 0.129 & 135.0 & 0.0 & 0.362 \\
$0.381 \mathrm{E}-02$ & $0.256 \mathrm{E}-03$ & 0.164 & 0.131 & 0.098 & 0.146 & 120.0 & 0.0 & 0.358 \\
$0.381 \mathrm{E}-02$ & $0.255 \mathrm{E}-03$ & 0.161 & 0.130 & 0.097 & 0.151 & 105.0 & 0.0 & 0.357 \\
$0.381 \mathrm{E}-02$ & $0.255 \mathrm{E}-03$ & 0.161 & 0.130 & 0.097 & 0.153 & 90.0 & 0.0 & 0.357 \\
$0.381 \mathrm{E}-02$ & $0.256 \mathrm{E}-03$ & 0.161 & 0.130 & 0.097 & 0.151 & 75.0 & 0.0 & 0.358 \\
$0.381 \mathrm{E}-02$ & $0.256 \mathrm{E}-03$ & 0.163 & 0.131 & 0.098 & 0.146 & 60.0 & 0.0 & 0.359 \\
$0.380 \mathrm{E}-02$ & $0.256 \mathrm{E}-03$ & 0.175 & 0.137 & 0.100 & 0.129 & 45.0 & 0.0 & 0.363 \\
$0.380 \mathrm{E}-02$ & $0.256 \mathrm{E}-03$ & 0.189 & 0.144 & 0.102 & 0.113 & 30.0 & 0.0 & 0.369 \\
$0.380 \mathrm{E}-02$ & $0.257 \mathrm{E}-03$ & 0.192 & 0.145 & 0.103 & 0.106 & 15.0 & 0.0 & 0.375 \\
$0.380 \mathrm{E}-02$ & $0.257 \mathrm{E}-03$ & 0.184 & 0.141 & 0.101 & 0.110 & 0.0 & 0.0 & 0.379 \\
$0.381 \mathrm{E}-02$ & $0.256 \mathrm{E}-03$ & 0.267 & 0.141 & 0.099 & 0.081 & 165.0 & 0.080 & 0.331 \\
$0.381 \mathrm{E}-02$ & $0.256 \mathrm{E}-03$ & 0.209 & 0.130 & 0.096 & 0.115 & 150.0 & 0.042 & 0.327 \\
$0.382 \mathrm{E}-02$ & $0.255 \mathrm{E}-03$ & 0.180 & 0.129 & 0.092 & 0.141 & 135.0 & 0.030 & 0.324 \\
$0.384 \mathrm{E}-02$ & $0.255 \mathrm{E}-03$ & 0.163 & 0.128 & 0.090 & 0.155 & 120.0 & 0.025 & 0.321 \\
$0.383 \mathrm{E}-02$ & $0.255 \mathrm{E}-03$ & 0.155 & 0.127 & 0.093 & 0.160 & 105.0 & 0.023 & 0.322 \\
$0.382 \mathrm{E}-02$ & $0.256 \mathrm{E}-03$ & 0.154 & 0.116 & 0.094 & 0.177 & 90.0 & 0.022 & 0.323 \\
$0.383 \mathrm{E}-02$ & $0.255 \mathrm{E}-03$ & 0.158 & 0.115 & 0.093 & 0.175 & 75.0 & 0.023 & 0.322 \\
$0.383 \mathrm{E}-02$ & $0.255 \mathrm{E}-03$ & 0.162 & 0.129 & 0.092 & 0.153 & 60.0 & 0.025 & 0.321 \\
$0.383 \mathrm{E}-02$ & $0.255 \mathrm{E}-03$ & 0.177 & 0.128 & 0.091 & 0.142 & 45.0 & 0.031 & 0.321 \\
$0.383 \mathrm{E}-02$ & $0.255 \mathrm{E}-03$ & 0.208 & 0.133 & 0.093 & 0.116 & 30.0 & 0.045 & 0.322 \\
$0.382 \mathrm{E}-02$ & $0.256 \mathrm{E}-03$ & 0.274 & 0.146 & 0.097 & 0.080 & 15.0 & 0.090 & 0.326 \\
\hline
\end{tabular}

Table 4. Same as Table 3 but with $\alpha^{B}=20, x_{0}^{B}=0.884, y_{0}^{B}=0.489, z_{0}^{B}=0.375 h_{\mathrm{r}}=2.5, h_{z}=0.156$. The first set of results have been obtained with $x_{1}$ fixed to 0 . In the second set of results $x_{1}$ is a free parameter.

\begin{tabular}{lllllllll}
\hline \hline Bulge & Disk & \multicolumn{7}{c}{ Bar } \\
$\rho_{0}^{B}$ & $\rho_{0}^{D}$ & $x_{0}^{b}$ & $\begin{array}{l}y_{0}^{b} \\
\text { kpc }\end{array}$ & $z_{0}^{b}$ & $\rho_{0}^{b}$ & $\begin{array}{l}\alpha^{b} \\
\text { kpc }\end{array}$ & $x_{1}$ & $\chi^{2}$ \\
\hline $0.474 \mathrm{E}-02$ & $0.256 \mathrm{E}-03$ & 0.201 & 0.151 & 0.102 & 0.101 & 165.0 & 0.0 & 0.374 \\
$0.475 \mathrm{E}-02$ & $0.256 \mathrm{E}-03$ & 0.195 & 0.148 & 0.101 & 0.110 & 150.0 & 0.0 & 0.370 \\
$0.475 \mathrm{E}-02$ & $0.256 \mathrm{E}-03$ & 0.180 & 0.141 & 0.099 & 0.127 & 135.0 & 0.0 & 0.366 \\
$0.476 \mathrm{E}-02$ & $0.255 \mathrm{E}-03$ & 0.171 & 0.137 & 0.097 & 0.140 & 120.0 & 0.0 & 0.364 \\
$0.476 \mathrm{E}-02$ & $0.255 \mathrm{E}-03$ & 0.168 & 0.135 & 0.096 & 0.147 & 105.0 & 0.0 & 0.364 \\
$0.476 \mathrm{E}-02$ & $0.255 \mathrm{E}-03$ & 0.166 & 0.134 & 0.096 & 0.150 & 90.0 & 0.0 & 0.364 \\
$0.476 \mathrm{E}-02$ & $0.255 \mathrm{E}-03$ & 0.168 & 0.135 & 0.096 & 0.147 & 75.0 & 0.0 & 0.364 \\
$0.476 \mathrm{E}-02$ & $0.255 \mathrm{E}-03$ & 0.171 & 0.137 & 0.097 & 0.140 & 60.0 & 0.0 & 0.365 \\
$0.476 \mathrm{E}-02$ & $0.256 \mathrm{E}-03$ & 0.179 & 0.141 & 0.098 & 0.128 & 45.0 & 0.0 & 0.367 \\
$0.475 \mathrm{E}-02$ & $0.256 \mathrm{E}-03$ & 0.193 & 0.148 & 0.101 & 0.112 & 30.0 & 0.0 & 0.371 \\
$0.474 \mathrm{E}-02$ & $0.256 \mathrm{E}-03$ & 0.198 & 0.150 & 0.101 & 0.104 & 15.0 & 0.0 & 0.375 \\
$0.475 \mathrm{E}-02$ & $0.257 \mathrm{E}-03$ & 0.196 & 0.149 & 0.101 & 0.104 & 0.0 & 0.0 & 0.379 \\
$0.474 \mathrm{E}-02$ & $0.256 \mathrm{E}-03$ & 0.283 & 0.141 & 0.100 & 0.778 & 165.0 & 0.080 & 0.341 \\
$0.477 \mathrm{E}-02$ & $0.256 \mathrm{E}-03$ & 0.213 & 0.134 & 0.095 & 0.115 & 150.0 & 0.042 & 0.333 \\
$0.477 \mathrm{E}-02$ & $0.255 \mathrm{E}-03$ & 0.189 & 0.123 & 0.094 & 0.142 & 135.0 & 0.030 & 0.333 \\
$0.480 \mathrm{E}-02$ & $0.255 \mathrm{E}-03$ & 0.166 & 0.131 & 0.088 & 0.155 & 120.0 & 0.025 & 0.332 \\
$0.478 \mathrm{E}-02$ & $0.256 \mathrm{E}-03$ & 0.158 & 0.126 & 0.092 & 0.164 & 105.0 & 0.022 & 0.332 \\
$0.478 \mathrm{E}-02$ & $0.255 \mathrm{E}-03$ & 0.159 & 0.129 & 0.092 & 0.161 & 90.0 & 0.022 & 0.332 \\
$0.479 \mathrm{E}-02$ & $0.255 \mathrm{E}-03$ & 0.162 & 0.118 & 0.090 & 0.175 & 75.0 & 0.023 & 0.332 \\
$0.478 \mathrm{E}-02$ & $0.256 \mathrm{E}-03$ & 0.164 & 0.133 & 0.092 & 0.151 & 60.0 & 0.025 & 0.331 \\
$0.477 \mathrm{E}-02$ & $0.256 \mathrm{E}-03$ & 0.187 & 0.121 & 0.095 & 0.144 & 45.0 & 0.032 & 0.333 \\
$0.477 \mathrm{E}-02$ & $0.256 \mathrm{E}-03$ & 0.221 & 0.132 & 0.095 & 0.113 & 30.0 & 0.045 & 0.333 \\
$0.475 \mathrm{E}-02$ & $0.256 \mathrm{E}-03$ & 0.283 & 0.147 & 0.099 & 0.780 & 15.0 & 0.089 & 0.337 \\
\hline
\end{tabular}


N. J. Rodriguez-Fernandez and F. Combes: Gas flow models in the Milky Way embedded bars, Online Material p 2

Table 5. Same as Table 3 but with $\alpha^{B}=35, x_{0}^{B}=0.774, y_{0}^{B}=0.429, z_{0}^{B}=0.382 h_{\mathrm{r}}=2.5, h_{z}=0.158$. The first set of results have been obtained with $x_{1}$ fixed to 0 . In the second set of results $x_{1}$ is a free parameter.

\begin{tabular}{lllllllll}
\hline \hline Bulge & Disk & \multicolumn{7}{c}{ Bar } \\
$\rho_{0}^{B}$ & $\rho_{0}^{D}$ & $x_{0}^{b}$ & $\begin{array}{l}y_{0}^{b} \\
\text { kpc }\end{array}$ & $z_{0}^{b}$ & $\rho_{0}^{b}$ & $\alpha^{b}$ & $x_{1}$ & $\chi^{2}$ \\
& & kpc & kpc & & deg & kpc & \\
\hline $0.619 \mathrm{E}-02$ & $0.256 \mathrm{E}-03$ & 0.205 & 0.153 & 0.102 & 0.100 & 165.0 & 0.0 & 0.382 \\
$0.619 \mathrm{E}-02$ & $0.256 \mathrm{E}-03$ & 0.197 & 0.150 & 0.101 & 0.110 & 150.0 & 0.0 & 0.377 \\
$0.620 \mathrm{E}-02$ & $0.256 \mathrm{E}-03$ & 0.182 & 0.143 & 0.099 & 0.126 & 135.0 & 0.0 & 0.374 \\
$0.621 \mathrm{E}-02$ & $0.256 \mathrm{E}-03$ & 0.172 & 0.138 & 0.097 & 0.141 & 120.0 & 0.0 & 0.371 \\
$0.621 \mathrm{E}-02$ & $0.255 \mathrm{E}-03$ & 0.169 & 0.136 & 0.097 & 0.147 & 105.0 & 0.0 & 0.371 \\
$0.621 \mathrm{E}-02$ & $0.255 \mathrm{E}-03$ & 0.168 & 0.136 & 0.096 & 0.149 & 90.0 & 0.0 & 0.371 \\
$0.621 \mathrm{E}-02$ & $0.255 \mathrm{E}-03$ & 0.169 & 0.136 & 0.097 & 0.147 & 75.0 & 0.0 & 0.372 \\
$0.621 \mathrm{E}-02$ & $0.256 \mathrm{E}-03$ & 0.172 & 0.137 & 0.097 & 0.141 & 60.0 & 0.0 & 0.373 \\
$0.620 \mathrm{E}-02$ & $0.256 \mathrm{E}-03$ & 0.182 & 0.142 & 0.099 & 0.126 & 45.0 & 0.0 & 0.375 \\
$0.619 \mathrm{E}-02$ & $0.256 \mathrm{E}-03$ & 0.196 & 0.149 & 0.101 & 0.110 & 30.0 & 0.0 & 0.379 \\
$0.619 \mathrm{E}-02$ & $0.257 \mathrm{E}-03$ & 0.202 & 0.151 & 0.102 & 0.102 & 15.0 & 0.0 & 0.383 \\
$0.619 \mathrm{E}-02$ & $0.257 \mathrm{E}-03$ & 0.201 & 0.151 & 0.102 & 0.101 & 0.0 & 0.0 & 0.386 \\
$0.605 \mathrm{E}-02$ & $0.252 \mathrm{E}-03$ & 0.305 & 0.144 & 0.100 & 0.073 & 165.0 & 0.079 & 0.353 \\
$0.608 \mathrm{E}-02$ & $0.252 \mathrm{E}-03$ & 0.218 & 0.135 & 0.097 & 0.111 & 150.0 & 0.042 & 0.350 \\
$0.608 \mathrm{E}-02$ & $0.252 \mathrm{E}-03$ & 0.188 & 0.126 & 0.095 & 0.141 & 135.0 & 0.030 & 0.348 \\
$0.609 \mathrm{E}-02$ & $0.252 \mathrm{E}-03$ & 0.170 & 0.126 & 0.094 & 0.157 & 120.0 & 0.025 & 0.348 \\
$0.610 \mathrm{E}-02$ & $0.252 \mathrm{E}-03$ & 0.162 & 0.122 & 0.093 & 0.171 & 105.0 & 0.022 & 0.348 \\
$0.610 \mathrm{E}-02$ & $0.252 \mathrm{E}-03$ & 0.163 & 0.119 & 0.091 & 0.178 & 90.0 & 0.022 & 0.348 \\
$0.611 \mathrm{E}-02$ & $0.252 \mathrm{E}-03$ & 0.164 & 0.124 & 0.089 & 0.171 & 75.0 & 0.022 & 0.349 \\
$0.611 \mathrm{E}-02$ & $0.252 \mathrm{E}-03$ & 0.173 & 0.127 & 0.091 & 0.158 & 60.0 & 0.025 & 0.349 \\
$0.609 \mathrm{E}-02$ & $0.252 \mathrm{E}-03$ & 0.193 & 0.126 & 0.095 & 0.140 & 45.0 & 0.031 & 0.348 \\
$0.609 \mathrm{E}-02$ & $0.252 \mathrm{E}-03$ & 0.222 & 0.137 & 0.095 & 0.112 & 30.0 & 0.044 & 0.348 \\
$0.606 \mathrm{E}-02$ & $0.252 \mathrm{E}-03$ & 0.308 & 0.149 & 0.100 & 0.073 & 15.0 & 0.089 & 0.351 \\
\hline
\end{tabular}

Table 6. Same as Table 3 but with $\alpha^{B}=45, x_{0}^{B}=0.693, y_{0}^{B}=0.367, z_{0}^{B}=0.385 h_{\mathrm{r}}=2.5, h_{z}=0.159$. The first set of results have been obtained with $x_{1}$ fixed to 0 . In the second set of results $x_{1}$ is a free parameter.

\begin{tabular}{lllllllll}
\hline \hline Bulge & Disk & \multicolumn{7}{c}{ Bar } \\
$\rho_{0}^{B}$ & $\rho_{0}^{D}$ & $x_{0}^{b}$ & $y_{0}^{b}$ & $z_{0}^{b}$ & $\rho_{0}^{b}$ & $\alpha^{b}$ & $x_{1}$ & $\chi^{2}$ \\
& & $\mathrm{kpc}$ & $\mathrm{kpc}$ & $\mathrm{kpc}$ & & $\mathrm{deg}$ & $\mathrm{kpc}$ & \\
\hline $0.761 \mathrm{E}-02$ & $0.255 \mathrm{E}-03$ & 0.211 & 0.167 & 0.105 & 0.095 & 165.0 & 0.0 & 0.389 \\
$0.763 \mathrm{E}-02$ & $0.255 \mathrm{E}-03$ & 0.204 & 0.154 & 0.103 & 0.106 & 150.0 & 0.0 & 0.382 \\
$0.766 \mathrm{E}-02$ & $0.255 \mathrm{E}-03$ & 0.184 & 0.143 & 0.099 & 0.126 & 135.0 & 0.0 & 0.378 \\
$0.767 \mathrm{E}-02$ & $0.256 \mathrm{E}-03$ & 0.173 & 0.138 & 0.097 & 0.141 & 120.0 & 0.0 & 0.376 \\
$0.767 \mathrm{E}-02$ & $0.255 \mathrm{E}-03$ & 0.170 & 0.136 & 0.097 & 0.147 & 105.0 & 0.0 & 0.375 \\
$0.767 \mathrm{E}-02$ & $0.255 \mathrm{E}-03$ & 0.168 & 0.136 & 0.096 & 0.149 & 90.0 & 0.0 & 0.376 \\
$0.767 \mathrm{E}-02$ & $0.255 \mathrm{E}-03$ & 0.170 & 0.136 & 0.097 & 0.147 & 75.0 & 0.0 & 0.376 \\
$0.767 \mathrm{E}-02$ & $0.256 \mathrm{E}-03$ & 0.172 & 0.138 & 0.097 & 0.141 & 60.0 & 0.0 & 0.377 \\
$0.766 \mathrm{E}-02$ & $0.255 \mathrm{E}-03$ & 0.183 & 0.143 & 0.099 & 0.126 & 45.0 & 0.0 & 0.379 \\
$0.763 \mathrm{E}-02$ & $0.256 \mathrm{E}-03$ & 0.204 & 0.151 & 0.102 & 0.107 & 30.0 & 0.0 & 0.384 \\
$0.761 \mathrm{E}-02$ & $0.256 \mathrm{E}-03$ & 0.217 & 0.161 & 0.106 & 0.092 & 15.0 & 0.0 & 0.391 \\
$0.764 \mathrm{E}-02$ & $0.257 \mathrm{E}-03$ & 0.204 & 0.153 & 0.102 & 0.099 & 0.0 & 0.0 & 0.390 \\
$0.766 \mathrm{E}-02$ & $0.255 \mathrm{E}-03$ & 0.288 & 0.150 & 0.098 & 0.076 & 165.0 & 0.079 & 0.350 \\
$0.767 \mathrm{E}-02$ & $0.255 \mathrm{E}-03$ & 0.220 & 0.134 & 0.096 & 0.112 & 150.0 & 0.042 & 0.347 \\
$0.768 \mathrm{E}-02$ & $0.255 \mathrm{E}-03$ & 0.186 & 0.126 & 0.096 & 0.141 & 135.0 & 0.030 & 0.347 \\
$0.770 \mathrm{E}-02$ & $0.255 \mathrm{E}-03$ & 0.166 & 0.134 & 0.092 & 0.151 & 120.0 & 0.025 & 0.345 \\
$0.771 \mathrm{E}-02$ & $0.255 \mathrm{E}-03$ & 0.163 & 0.122 & 0.090 & 0.171 & 105.0 & 0.022 & 0.346 \\
$0.771 \mathrm{E}-02$ & $0.255 \mathrm{E}-03$ & 0.160 & 0.119 & 0.090 & 0.178 & 90.0 & 0.022 & 0.346 \\
$0.771 \mathrm{E}-02$ & $0.255 \mathrm{E}-03$ & 0.165 & 0.122 & 0.090 & 0.170 & 75.0 & 0.022 & 0.346 \\
$0.770 \mathrm{E}-02$ & $0.255 \mathrm{E}-03$ & 0.171 & 0.122 & 0.092 & 0.162 & 60.0 & 0.025 & 0.346 \\
$0.769 \mathrm{E}-02$ & $0.255 \mathrm{E}-03$ & 0.193 & 0.126 & 0.094 & 0.140 & 45.0 & 0.031 & 0.346 \\
$0.768 \mathrm{E}-02$ & $0.255 \mathrm{E}-03$ & 0.227 & 0.133 & 0.095 & 0.112 & 30.0 & 0.045 & 0.347 \\
$0.765 \mathrm{E}-02$ & $0.256 \mathrm{E}-03$ & 0.308 & 0.146 & 0.100 & 0.073 & 15.0 & 0.090 & 0.350 \\
\hline
\end{tabular}

